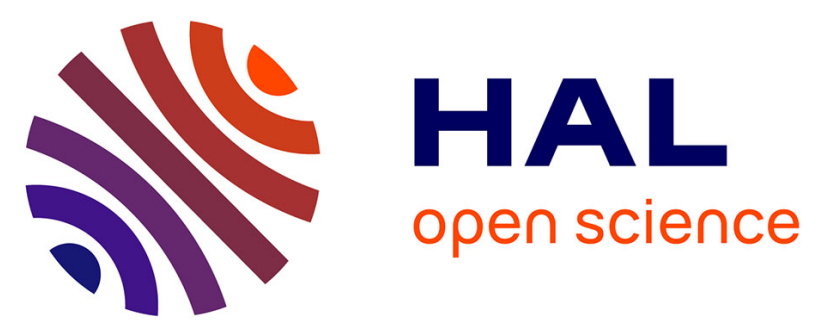

\title{
A method for the quantitative extraction of gold nanoparticles from human bronchoalveolar lavage fluids through a glycerol gradient
}

Dimitrios Bitounis, Vincent Barnier, Cyril Guibert, Jérémie Pourchez, Valérie Forest, Delphine Boudard, Jean-François Hochepied, Pierre Chelle, Jean-Michel Vergnon, Michèle Cottier

\section{To cite this version:}

Dimitrios Bitounis, Vincent Barnier, Cyril Guibert, Jérémie Pourchez, Valérie Forest, et al.. A method for the quantitative extraction of gold nanoparticles from human bronchoalveolar lavage fluids through a glycerol gradient. Nanoscale, 2018, 10 (6), pp.2955-2969. 10.1039/C7NR04484D . hal-01806118

HAL Id: hal-01806118

https://hal-mines-paristech.archives-ouvertes.fr/hal-01806118

Submitted on 16 Oct 2018

HAL is a multi-disciplinary open access archive for the deposit and dissemination of scientific research documents, whether they are published or not. The documents may come from teaching and research institutions in France or abroad, or from public or private research centers.
L'archive ouverte pluridisciplinaire HAL, est destinée au dépôt et à la diffusion de documents scientifiques de niveau recherche, publiés ou non, émanant des établissements d'enseignement et de recherche français ou étrangers, des laboratoires publics ou privés. 


\section{A method for the quantitative extraction of gold nanoparticles from human bronchoalveolar lavage fluids through a glycerol gradient}

Dimitrios Bitounis ${ }^{\mathrm{a}}$, Vincent Barnier ${ }^{\mathrm{b}}$, Cyril Guibert ${ }^{\mathrm{c}}$, Jérémie Pourchez ${ }^{\mathrm{d}, \mathrm{e}, \mathrm{f}}$, Valérie Forest $^{\text {d,e,f, }}$, Delphine Boudard ${ }^{\mathrm{a}, \mathrm{g}}$, Jean-François Hochepied ${ }^{\text {h }}$, Pierre Chelle ${ }^{\mathrm{d}, \mathrm{e}, \mathrm{f}}$ Jean-Michel $^{-}$ Vergnon $^{\mathrm{a}, \mathrm{c}}$ and Michèle Cottier ${ }^{\mathrm{a}}$

${ }^{a}$ Université de Lyon, Faculté de Médecine, INSERM U1059 SAINBIOSE, F-42270 SaintEtienne, France. E-mail: dimitrios.bitounis@univ-st-etienne.fr

${ }^{\mathrm{b}}$ Ecole Nationale Supérieure des Mines, SMS-EMSE, CNRS:UMR5307, LGF, F-42023 SaintEtienne, France

${ }^{\mathrm{c}}$ CHU Saint-Etienne, Department of Chest Diseases and Thoracic Oncology, F-42055 SaintEtienne, France

${ }^{\mathrm{d}}$ Ecole Nationale Supérieure des Mines de Saint-Etienne, CIS-EMSE, SAINBIOSE, F-42023 Saint Etienne, France

${ }^{\text {e } I N S E R M, ~ U 1059, ~ F-42023 ~ S a i n t-E t i e n n e, ~ F r a n c e ~}$

${ }^{\mathrm{f}}$ Université de Lyon, F-69000 Lyon, France

${ }^{\mathrm{g}}$ CHU de Saint-Etienne, UF6725 Laboratoire de Cytologie et Histologie Rénale, F-42055 SaintEtienne, France

${ }^{\text {h }}$ MINES ParisTech, PSL Research University, MAT - Centre des matériaux, CNRS UMR 7633, BP 8791003 Evry, France 


\begin{abstract}
Bronchoalveolar lavage (BAL) is a diagnostic procedure which samples the cellular and noncellular components of the pulmonary epithelial surface. The inherent biological noise of BAL fluids inhibits their direct mineralogical analysis while currently available particle retrieval protocols are suspected to impose quantitative and qualitative bias on the studied particle load. This study presents a simple method for the near-lossless extraction of citrate-capped gold nanoparticles from human BAL fluids at sub-ppm levels which enables their quantitation and surface characterization. This procedure was modeled according to fundamental principles of particle sedimentation and liquid-liquid interdiffusion and was evaluated by a battery of analytical techniques. The extraction yield of gold nanoparticles ranged from 61 to $86 \%$, with a quantitation limit at $0.5 \mu \mathrm{g} \mathrm{ml}^{-1}$, as measured by inductively-coupled optical emission spectroscopy. Dynamic light scattering could resolve the hydrodynamic size distribution of extracted particles which returned significantly different photon count rates at various concentrations. Their shape and primary size were easily observable by electron microscopy while atomic force microscopy, Auger electron spectroscopy and X-ray photoelectron spectroscopy could respectively probe the particles' biomolecular corona, detect surfaceadsorbed S- and N- species, and identify carbon-based covalent bonds.
\end{abstract}




\section{Introduction}

The nanosized particle load of human clinical samples cannot be extensively studied due to technical limitations. This knowledge gap has been characterized by the U.S. National Science and Technology Council as an area that demands further research. ${ }^{1}$ In a similar vein, the European Union has launched a project workshop ("NanoChOp") for the improvement of chemical and optical characterization of nanomaterials in biological systems. ${ }^{2}$ In the clinic, several teams have already identified the need to detect possibly harmful nanoparticles in human biological samples, like Pelclova et al. and Song et al. who investigated exhaled breath condensates and pleural fluids from exposed individuals, respectively. ${ }^{3,4} \mathrm{~A}$ review of the available literature has revealed that clinical cases of nanotoxicological interest face pitfalls which might hinder the quantification and qualitative characterization of detected nanoparticles. ${ }^{5}$ This work focuses on the extraction and characterization of gold nanoparticles from bronchoalveolar lavage (BAL) fluids. During BAL, cellular and non-cellular components of the epithelial surface of the terminal bronchioles and alveoli are sampled by intrapulmonary instillation of sterile saline solution. ${ }^{6}$ The cytological and biochemical profile of aspirated fluids complement other diagnostic tools (e.g., radiographs) and can narrow down the differential diagnosis of various conditions, like interstitial lung diseases and infectious pneumonias. BAL is generally well-tolerated and safer than lung biopsies, but is only performed when there are serious indications and is thus considered an invasive technique.

Traditionally, mineralogical analyses of human BAL fluids required the oxidation of their biological contents and filtration of the liquid digest for the observation of the solid parts of the retentate by optical and/or electron microscopy. ${ }^{7}$ Such procedures have been applied with little variation for more than three decades in order to analyze coarse and fine, metal and carbonbased particles ${ }^{8,9}$ as well as asbestos fibres and carbon nanotubes ${ }^{10,11}$ in pulmonary lavages. Nevertheless, the quantitative retrieval of nanosized metal and metal oxide particles from human BAL fluids has not yet been described, despite their suspected pathogenic potential. The use of analytical ultracentrifugation (AUC) has been recently rekindled and it can provide the means for the fractionation of nanoparticles even in complex media, as shown with the isolation of exosomes from a cell culture suspension by Livshits et al. ${ }^{12}$ However, the cost of acquisition, operational complexity, and maintenance of an ultracentrifuge have stymied its widespread adoption. Single particle inductively-coupled plasma mass spectroscopy (spICP-MS) is another extremely sensitive technique that allows for the simultaneous elemental analysis and particle size characterization of liquid biological samples, as showcased by Witzler et al. for the measurement of gold and silver nanoparticles in human whole blood. ${ }^{13}$ Future advancements 
should render spICP-MS more approachable to clinicians, but, for the time being, it remains a relatively expensive technique.

The currently proposed method is clinically-oriented in that it only requires typical benchtop equipment. Its objective is to promote the lossless extraction of gold nanoparticles from human BAL fluids so that particles can be subsequently analyzed by both bulk and nanoscale characterisation techniques. The biological matrix used for the development of this method is bronchial washing fluid which is representative of the final conducting airways. In fact, particlerelated injury in the terminal bronchioles ${ }^{14}$ and modeled deposition patterns in non-healthy human lungs suggest that most nanoparticles do not reach the alveolar sacs. ${ }^{15}$ Being the first aspirations obtained by BAL, bronchial washing fluids contain more mucus and epithelial cells than subsequent aliquots ${ }^{16}$ and thus pose a greater analytical challenge for the extraction and characterization of small particles. Gold nanoparticles were used as analytes because they facilitated the assessment of the method's sensitivity. Moreover, gold nanoparticles have dominated the engineered nanoparticle market and have become the staple material in many scientific fields for the development of nano-enabled applications.

\section{Materials and methods}

\subsection{Theoretical background and model development}

The method is completed in two consecutive steps: Step I is a density barrier centrifugation for the removal of coarse particles and Step II is a gradient centrifugation for the extraction of the remaining, smaller particles. The description of both steps is based on principles of sedimentation and diffusion. In Table 1, some assumptions regarding the size, shape, and density of particles as well as their liquid milieu are listed. The method will be described with the set of materials and volumes employed during its development: $2 \mathrm{ml}$ Protein LoBind ${ }^{\mathrm{TM}}$ tubes (Eppendorf $\left.{ }^{\circledR}\right)$, a Heraeus ${ }^{\mathrm{TM}}$ Megafuge $^{\mathrm{TM}} 16$ with the TX-400 swinging-bucket rotor (ThermoFisher Scientific ${ }^{\mathrm{TM}}$ ), 22G $1^{1 / 2}$ Terumo® needles mounted on $2 \mathrm{ml}$ syringes, glycerol (extra-pure, Acros Organics ${ }^{\mathrm{TM}}$ ), and sodium hydroxide $(\mathrm{NaOH})$ (analytical grade, Merck $\mathrm{KGaA}$ ). Manual experiments and centrifugations were performed at room temperature and $0^{\circ} \mathrm{C}$, respectively.

\subsubsection{Step I: isolation of coarse particles.}

Step I aims at segregating coarse particles expected to be found in bronchial washing fluids: $0.50 \mathrm{ml}$ of glycerol-based cushion $\left(75 \% \mathrm{v} / \mathrm{v}\right.$ glycerol in $1 \mathrm{mM} \mathrm{NaOH}$, density $\rho_{\mathrm{g}}=1.210 \mathrm{~g}$ $\mathrm{cm}^{-3}$ ), referred to as "cushion", are slowly injected under $1.00 \mathrm{ml}$ of the less dense bronchial washing fluid and subjected to centrifugation. For short periods of time at low temperature the 
two compartments do not mix, thus Step I can be described as a density barrier centrifugation. Several hundred to a few thousand $\mathrm{g}\left(\mathrm{m} \mathrm{s}^{-2}\right)\left(\mathrm{rcf}_{1}\right)$ allow coarse particles to penetrate the cushion (Fig. 1A). As established elsewhere, ${ }^{17}$ Stokes law can be used to describe the movement of spherical nanoparticles with radius $\mathrm{r}_{\mathrm{P}}(\mathrm{m})$ and density $\rho_{\mathrm{P}}$ over time $\mathrm{t} 1 \mathrm{(s)}$ in a less dense medium $\left(\rho_{\mathrm{g}}\right)$ :

$$
\mathrm{d}_{1}=2 \mathrm{rP}^{2}\left(\rho \mathrm{P}-\rho_{\mathrm{g}}\right) \operatorname{rcf}_{1} \mathrm{gt}_{1}(9 \eta)^{-1}
$$

where $d_{1}$ is the distance traveled in the cushion $(\mathrm{m}), \mathrm{g}$ is the gravitational acceleration $(9.8 \mathrm{~m}$ $\mathrm{s}^{2}$ ), and $\eta$ is the cushion's dynamic viscosity $\left(\mathrm{kg} \mathrm{m}^{1} \mathrm{~s}^{1}\right)$. Coarse particles with sedimentation coefficients $\mathrm{s}>\mathrm{d}_{1} \mathrm{rcf}_{1}^{-1} \mathrm{~g}^{-1} \mathrm{t}_{1}^{-1}$ move below $\mathrm{d}_{1}$, whereas particles that have traveled $<\mathrm{d}_{1}$ are collected by puncturing the tube and aspirating the volume supernatant to the $\mathrm{d}_{1}$ plane (Fig. 1B). The volume of the collected aliquot $\mathrm{Val}(\mathrm{ml})$ consists of the original bronchial washing fluid and part of the cushion:

$$
\mathrm{Val}=1.00+\left(0.50-\pi \mathrm{r}_{\mathrm{tb}^{2}} \mathrm{~d}_{1} \times 10^{6}\right)
$$

where $\mathrm{r}_{\mathrm{tb}}$ is the tube's radius (here, $\mathrm{r}_{\mathrm{tb}}=5 \times 10^{-3} \mathrm{~m}$ ).

\subsubsection{Step II: extraction of nanoparticles.}

The aliquot collected in Step I is transferred to a new tube and $0.50 \mathrm{ml}$ of cushion is slowly injected beneath it. Similarly to the previous step, as soon as the aliquot and cushion come into contact, they begin to mix, but the extraction of small particles at low centrifugal forces requires significantly longer durations. Consequently, their mixing is no longer negligible and needs to be described: driven by the concentration difference, glycerol molecules from the area of high concentration (cushion) diffuse into the area of low concentration (aliquot) (Fig. 1C). The temporal, one-dimensional evolution of glycerol's concentration $\mathrm{C}\left(\mathrm{mol} \mathrm{L}^{-1}\right)$ is given by a solution to Fick's second law for the non-steady-state inter-diffusion between two semi-infinite liquids brought together at a plane. ${ }^{18,19}$ Here, this plane is the initial aliquot-cushion interface, where $\mathrm{x}=0$. Specifically, it is:

$\mathrm{C}(\mathrm{x}, \mathrm{t})=\frac{\mathrm{Cgc}+\mathrm{Cal}}{2}+\frac{\mathrm{Cgc}-\mathrm{Cal}}{2} \operatorname{erf} \frac{\mathrm{x}}{\sqrt{\mathrm{Dt}}}$

for $\mathrm{x}>0$ (arbitrarily set in the aliquot region), and

$C(x, t)=\frac{C g c+C a l}{2}-\frac{C g c-C a l}{2} \operatorname{erf} \frac{x}{\sqrt{D t}}$ 
for $\mathrm{x}<0$ (in the cushion region).

$\mathrm{Cal}$ and $\mathrm{Cgc}$ are the glycerol concentrations in the aliquot and cushion, respectively; $\mathrm{D}$ is the aliquot-cushion mutual diffusion coefficient $\left(\mathrm{m}^{2} \mathrm{~s}^{-1}\right)$ (Fig. 1D). Mutual diffusion coefficients for interdiffusing water-glycerol mixtures can be experimentally approximated. The method was developed with a pooled bronchial washing fluid (presented in section 2.2.1), for which the diffusion coefficient under the specific experimental conditions was $D=4.4 \times 10^{-11} \mathrm{~m}^{2} \mathrm{~s}^{-1}$. At a fixed temperature, the medium's density $(\rho)$ and viscosity $(\eta)$ depend on the continuously changing glycerol concentration. Furthermore, the relative centrifugal force applied on a sedimenting particle increases with the distance $\mathrm{R}$ from the axis of rotation, until it reaches a theoretical maximum value ( $\left.\mathrm{rcf}_{2}\right)$, characteristic of the rotor-tube combination; in this setup $\mathrm{rcf}_{2}$ $=4000$. The process of sedimentation was thus modeled to calculate the amount of time $t_{2}$ required for a particle of a given density and size to reach the part of the cushion that has not yet interdiffused with its supernatant volume and distance itself an additional $1 \mathrm{~mm}$ below the expanding glycerol gradient. A linear regression for $\rho$ was extrapolated from the Handbook of Chemistry and Physics ${ }^{20}$ and $\eta$ was calculated according to a formula proposed by Cheng. ${ }^{21}$ More information on the diffusion coefficient, density, and viscosity can be found in File S1 of the Supplementary Information. The model was scripted in Matlab and is made available by the authors upon request. At the end of Step II, the extent of diffusion $\mathrm{d}_{2}(\mathrm{~m})$ is the same above and below the initial aliquot-cushion interface and can be approximated as:

$\mathrm{d}_{2} \approx \sqrt{7} \mathrm{Dt}_{2}$

where $t_{2}(s)$ is the previously calculated duration of Step II. This approximation is necessary because, according to eqn (4), the glycerol concentration tends to its original value as $t$ or $|x|$ tend to infinity. In this model, this approximation is reflected on the minimum glycerol concentration of the gradient that is arbitrarily set as "close enough" to the original glycerol concentration in the cushion. Here, the particle is considered "extracted" as soon as the glycerol concentration at its position is $\geq 97.5 \%$ of the cushion's original concentration (for more details, see File S1 of the Supplementary Information). The part of the cushion that contains the extracted particles has to be separated from the rest of the sample. This is achieved by collecting the liquid supernatant above the plane at $\mathrm{d}_{2}$ (sBW). Its volume, VsBW, consists of the initial aliquot volume Val, and a portion of the retrieved cushion:

$\mathrm{VsBW}=\mathrm{Val}+\left(0.50-\pi \mathrm{r}_{\mathrm{tb}}{ }^{2} \mathrm{~d}_{2} \times 10^{6}\right)$

The remaining volume of the cushion is the particle-containing fraction (pBW - see Fig. 1C) that lies below $\mathrm{d}_{2}$ and which should be collected with the addition of $1 \mathrm{mM} \mathrm{NaOH}$ to prevent 
the protonation of residual hypochlorite ions $\left(\mathrm{OCl}^{-}\right)$to the much more potent oxidant hypochlorous acid ( $\mathrm{HOCl})$.

\subsection{Biological matrix and gold nanoparticles}

2.2.1. Retrieval and conditioning of bronchial washing fluids.

BALs were performed on patients with symptoms of infiltrative pulmonary diseases with a flexible fiberoptic bronchoscope, in line with the official recommendations of the European Respiratory Society. ${ }^{22}$ Briefly, a local anesthetic (xylocaine) was sprayed on the oropharynx and vocal chords to facilitate the bronchoscope's passage towards the sub-segmental bronchi (middle lobe or lingula). When the bronchoscope reached the targeted bronchus, it was wedged in place and $50 \mathrm{ml}$ of tepid and sterile $0.9 \mathrm{w} / \mathrm{v} \mathrm{NaCl}$ solution were slowly injected. A fraction $(\approx 60 \%)$ of the injected volume was retrieved into plastic containers by low-pressure suctioning. This first aliquot is enriched with mucus and epithelial cells and is therefore better described as bronchial washing fluid. Five (5) $\mathrm{ml}$ of bronchial washing fluid from each patient were diluted with an equal volume of $0.0183 \mathrm{M} \mathrm{Na}$-hypochlorite $(\mathrm{NaOCl})$ solution (commercially available, technical grade) and stored in plastic $50 \mathrm{ml}$ tubes (BD Flacon) protected from light at $4{ }^{\circ} \mathrm{C}$. In order to develop and assess the methodology, multiple $\mathrm{NaOCl}$-treated bronchial washing fluids were pooled to create a reference bronchial washing fluid, hereafter referred to as BWF. Specifically, 50 individual samples were agitated and briefly sonicated in a 3-inch cup-horn sonicator (three $10 \mathrm{~s}$ bursts, at 70\% amplitude, Branson Ultrasonics), then $1 \mathrm{ml}$ aliquots from each were sampled to create a $50 \mathrm{ml}$ pool (to avoid selection bias, fluids were sampled in the order of patient's admission date) which was finally triple-filtered through $0.2 \mu \mathrm{m}$ polyethersulfone membranes (Merck Millipore ${ }^{\mathrm{TM}}$ ) to remove preexisting particles that could interfere with the development and evaluation of the extraction method.

\subsubsection{Characterisation of pooled bronchial washing fluid}

\subsubsection{UV spectrum and total protein content.}

Commercially available $\mathrm{NaOCl}$ solutions for the conditioning of bronchial washing fluids contain small amounts of surfactants. Therefore, it was replaced by Dulbecco's phosphatebuffered saline (DPBS) (Sigma-Aldrich@) in order to record the ultraviolet (UV) extinction spectrum and measure the total protein content of BWF: $4000 \mu$ were transferred in an Amicon ${ }^{\circledR}$ Ultra-4 centrifugal filter tube of $3 \mathrm{kDa}$ nominal molecular weight cut-off (Merck Millipore, Denmark) and were centrifuged at $2500 \mathrm{~g}$ for $40 \mathrm{~min}$ at $4{ }^{\circ} \mathrm{C}$. The filtrate was then reconstituted to $4000 \mu \mathrm{l}$ with DPBS. The total protein content was measured using the Pierce ${ }^{\mathrm{TM}}$ 
$660 \mathrm{~nm}$ Protein Assay kit (ThermoFisher Scientific ${ }^{\mathrm{TM}}$ ) according to the manufacturer's instructions: $10 \mu \mathrm{l}$ of reconstituted BWF were placed in a 96-well plate, $150 \mu \mathrm{l}$ of the Protein Assay Reagent were added and the plate was first shaken for $60 \mathrm{~s}$, then incubated for $5 \mathrm{~min}$. In this colorimetric assay, the sample's absorption in the presence of the reagent is measured at $660 \mathrm{~nm}$ using a microplate reader (Multiskan ${ }^{\mathrm{TM}} \mathrm{GO}$, ThermoFisher Scientific ${ }^{\mathrm{TM}}$ ). Total protein concentrations were extrapolated by comparison with a standard curve from bovine serum albumin samples. Finally, a quartz cuvette was used in the same instrument to record the UV extinction spectra of the reconstituted BWF at various dilutions.

\subsubsection{Reduction potential.}

Excluding any alveolar lining fluid or mucus retrieved during BAL, the continuous phase of $\mathrm{NaOCl}$-treated bronchial washings consists of a $50 \% \mathrm{v} / \mathrm{v}$ solution of $0.0183 \mathrm{M} \mathrm{NaOCl}$ in 0.9 w/v NaCl, hereafter referred to as blank BWF. This solution's $\mathrm{pH}$ was measured with a benchtop glass electrode $\left(\mathrm{pHenomenal}{ }^{\circledR} \mathrm{pH} 1000 \mathrm{~L}, \mathrm{VWR}\right)$ and its reduction potential $\mathrm{E}_{\mathrm{ClO}-}$ was calculated based on the following half-reaction (for more details, see File S1 of the Supplementary Information):

$\mathrm{ClO}^{-}+2 \mathrm{e}^{-}+\mathrm{H}_{2} \mathrm{O} \rightleftharpoons \mathrm{Cl}^{-}+2 \mathrm{OH}^{-}$

\subsubsection{Characterization of gold nanoparticles.}

Citratecapped, gold nanoparticles (AuNPs) dispersed in $0.1 \mathrm{mM}$ phosphate buffered solution (PBS) were purchased from Sigma-Aldrich $\odot$. The nominal concentration of the stock dispersionand the particles' geometric diameter were $1.9 \times 10^{10}$ particles per $\mathrm{ml}$ and $60 \mathrm{~nm}$, respectively. Their primary size distribution was calculated by using ImageJ (v. 2.0.0) using images obtained by transmission electron microscopy (TEM) (CM200, Philips) and their shape was observed by field-emission scanning electron microscopy (FESEM) (Supra 55VPTM, Carl Zeiss AG). Their z-potential was measured using a polycarbonate, folded, capillary cell (Malvern Instruments ${ }^{\mathrm{TM}}$ ).

\subsubsection{Setting the extraction parameters.}

One (1.00) $\mathrm{ml} \mathrm{BWF}$ aliquots were spiked with the following number of AuNPs (particles $\times$ $\left.10^{8}\right): 2.4,4.8,7.1,9.5$ and 19.0 . The samples were manually agitated, incubated at $37{ }^{\circ} \mathrm{C}$ for 40 min, and then subjected to the previously described Steps I \& II. The negative sample was BWF alone (without AuNPs). 
Although BWF had been originally filtered at $0.2 \mu \mathrm{m}$ to remove any native particles, Step I was still applied in order to assess how it might impact the final extraction of AuNPs. Samples were centrifuged at $\mathrm{rcf}_{1}=2500$ for $\mathrm{t}_{1}=10 \mathrm{~min}$. The tubes were then punctured at $3 \mathrm{~mm}$ beneath the BWF-cushion interface so that any particles with geometric diameters larger than $\approx 1.0 \mu \mathrm{m}$ would be left in the cushion, as per eqn (1). It needs to be mentioned that syringes were manually operated, so puncturing the tubes could only be precise down to the millimetre level. The volume that lied above the puncture point (i.e., the original BWF volume spiked with AuNPs and part of the cushion) was collected and transferred to the next step. In Step II, new cushions were slowly injected under the aliquots transferred from the previous step. The effective density of citrate-capped AuNPs was approximated at $17.055 \mathrm{~g} \mathrm{~cm}^{-3}$ (for more details, see File S1 of the Supplementary Information) and their smallest primary particle size was found to be $45 \mathrm{~nm}$ (presented in the Results section). As depicted in Fig. 2, the derived model describes how a single AuNP with the above-mentioned size and density would require 210 min to: travel the aliquot's entire length, cross the aliquot-cushion interdiffusion layer, and sink an additional $1 \mathrm{~mm}$ beneath the expanding glycerol gradient. Because the rotor requires several minutes to come to a halt, the final duration of Step II was $240 \mathrm{~min}$. According to eqn (5), the expected extent of interdiffusion after $240 \mathrm{~min}$ was $\mathrm{d}_{2}=2.1 \mathrm{~mm}$, at which distance below the initial aliquot-cushion interface tubes were punctured. The aspirated volume supernatant to the puncture point is referred to as sBWAu while the remaining cushion with the extracted particles was reconstituted to $1.00 \mathrm{ml}$ with the ad hoc addition of $1 \mathrm{mM} \mathrm{NaOH}$ and is referred to as pBWAu.

\subsection{Evaluation of the extraction method}

\subsubsection{Characterization of the glycerol cushion.}

To assess the glycerol content of sBWAu and pBWAu while avoiding interferences from biological residues, blank BWF samples were used as surrogates and were treated under the same conditions as BWF spiked with AuNPs. The total organic carbon (TOC) content of the volumes corresponding to sBWAu and pBWAu was measured at $240 \mathrm{~min}$ and at two time points preceding the completion of Step II (60 and $120 \mathrm{~min}$ ). In brief, samples were diluted in $0.1 \mathrm{M}$ hydrochloric acid and their organic carbon content was quantified by combustion at $850{ }^{\circ} \mathrm{C}$ in a Vario-TOC Cube (Elementar). Blank BWF and negative samples were treated according to the same particle extraction method and the retrieved cushions were analysed by Fourier transform infrared spectroscopy (FTIR) in order to detect qualitative changes caused by any contaminating biological residues. Multiple scans at $1 \mathrm{~cm}^{-1}$ resolution obtained by a MIR TF 
VERTEX 70 equipped with an ATR system were averaged to produce FTIR spectra. Finally, the total protein content of the cushion of negative samples was also measured according to the procedure described in 2.2.2.1.

\subsubsection{Particle extraction yield.}

The method's efficiency in extracting AuNPs from BWF was evaluated by means of ICP-OES. Traditionally, this technique requires the solid contents of a sample to be dissolved, however recent studies have shown that it can provide quantitative results when measuring undigested particles. ${ }^{23,24}$ This type of direct measurement requires the particles' geometric diameter to be less than $2 \mu \mathrm{m}$ (so that particles avoid exclusion by the instrument's nebulizer) and that they are stably dispersed long enough for their representative sampling. On these grounds, pBWAu aliquots $(0.40 \mathrm{ml})$ were diluted in DPBS $(4.60 \mathrm{ml})$ and their content in gold was assessed at $242.795 \mathrm{~nm}$ (Jy138 Ultrace, Jobin-Yvon). In order to measure the signal of AuNPs in a medium that would be compositionally similar to pBWAu, a matrix-matched calibration curve was plotted based on the previously presented TOC results. Specifically, AuNPs $(2.4,4.8,7.1,9.5$ and 19.0 particles $\times 10^{8}$ ) were dispersed in $1.00 \mathrm{ml}$ solutions of $3.7 \mathrm{M}$ glycerol and $0.001 \mathrm{mM}$ $\mathrm{NaOH}$. Then, $0.4 \mathrm{ml}$ aliquots were diluted in $4.60 \mathrm{ml}$ of DPBS and their light intensity signals were correlated to their particle concentration by linear regression. The signal intensities of pBWAu were compared against the calibration curve, and it was thus possible to evaluate the yield of the extraction method. Finally, the method's limit of blank and the limit of quantitation were calculated according to the EP17-A2 guidelines issued by the Clinical and Laboratory Standards Institute. ${ }^{25}$

\subsubsection{Hydrodynamic size of extracted particles.}

After a short cup-horn sonication (three $10 \mathrm{~s}$ bursts, at 70\% amplitude), BWF, AuNPs (in deionized water), pBWAu, and sBWAu were assessed by DLS (Zetasizer ${ }^{\mathrm{TM}}$ NanoZS, Malvern Instruments $\left.{ }^{\mathrm{TM}}\right)$. The instrument performed standard backscatter measurements at $175^{\circ}$ with a $633 \mathrm{~nm} \mathrm{He}-\mathrm{Ne}$ laser. Tests were performed at $25{ }^{\circ} \mathrm{C}$, on 100 or $1000 \mu \mathrm{l}$ aliquots using disposable micro-cuvettes made from optical polystyrene (Kartell S.p.A) or disposable polymethyl methacrylate cuvettes (MC2), respectively. The measurement position (distance from cuvette's wall) and power of the incident laser beam were set automatically by the instrument. Measurements were performed in triplicate (unless otherwise noted) with each measurement consisting of $12 \times 16$ runs of $10 \mathrm{~s}$. Results were then averaged using a built-in software tool and processed with the "multiple narrow modes" algorithm, suitable for heterogeneous samples. 
For AuNPs and BWF, the dispersant's viscosity and refractive index were that of pure water; for $\mathrm{pBWAu}$ and $\mathrm{sBWAu}$, these parameters were corrected according to their glycerol content as calculated by TOC measurements.

\subsubsection{Electron microscopy and surface analyses of extracted particles.}

For all electron microscopy and surface analyses, $100 \mu \mathrm{l}$ of stock or extracted AuNPs from the most concentrated sample were added in $1.00 \mathrm{ml}$ of deionized water, vortexed and centrifuged at $2500 \mathrm{~g}$ for $10 \mathrm{~min}$ at room temperature in a swinging-bucket rotor. Then, $1.00 \mathrm{ml}$ of supernatant liquid was slowly aspirated and the remaining volume was subjected to the same wash cycle twice more.

\subsubsection{FESEM, BSE, and TEM.}

To prepare flat and conducting surfaces, mica sheets were placed on aluminium-based specimen mounts and were coated with a $50 \mathrm{~nm}$ high-resolution carbon layer (682 precision coating system, Gatan Inc.). To minimize charge effects from the electron beam, the sheets were connected to the rims of the metal stubs with conductive tape. A small volume of sample (1-2 $\mu 1)$ was deposited on the coated mica surface and left to dry protected from dust. Images were obtained at a working distance of $4 \mathrm{~mm}$ using the angular selective back-scatter detector operated between 10 and $20 \mathrm{keV}$ and the in-lens secondary electron detector operated at $1 \mathrm{keV}$. The preparation steps are presented in Fig. S2 of the Supplementary Information. The morphology of the extracted particles was observed by TEM with a TECNAI 20FST microscope operating at $200 \mathrm{kV}$. The samples were left to dry protected from dust placed on a 200 mesh, carbon-coated Ni grid (Agar Scientific Ltd) and were then observed without the use of staining agents.

\subsubsection{X-Ray photoelectron spectroscopy (XPS) and Auger electron spectroscopy (AES).} XPS and AES analyses were performed with a Thermo VG Thetaprobe instrument. For the XPS analysis, a focused monochromatic $\mathrm{Al} \mathrm{K} \alpha$ source ( $\mathrm{hv}=1486.68 \mathrm{eV}, 400 \mu \mathrm{m}$ spot size) was used. Photoelectrons were analyzed using a concentric hemispherical analyzer operating in the constant $\Delta$ mode. The energy scale was calibrated with the sputter-cleaned pure reference samples of $\mathrm{Au}, \mathrm{Ag}$ and $\mathrm{Cu}$ so that the $\mathrm{Au} 4 \mathrm{f} 7 / 2, \mathrm{Ag} 3 \mathrm{~d} 5 / 2$ and $\mathrm{Cu} 2 \mathrm{p} 3 / 2$ were positioned at the binding energies of 83.98, 386.26 and $932.67 \mathrm{eV}$, respectively. Spectra were recorded in the binding energy range of $0-1300 \mathrm{eV}$ with a step size of $1 \mathrm{eV}$ and a pass energy of $300 \mathrm{eV}$ for survey scans and ranges of 80-96 eV for Au 4f, 96-108 eV for Si 2p, 527-537 eV for O 1s, 
281-291 eV for C 1s, and 394-412 eV for C 1s with a step size of $0.1 \mathrm{eV}$ and a pass energy of $50 \mathrm{eV}$ for narrow scans. The two pass energies give energy resolution (width of the $\mathrm{Ag} 3 \mathrm{~d} 5 / 2$ peak) measured on the sputter clean silver samples of respectively $1 \mathrm{eV}$ and $0.55 \mathrm{eV}$. Chemical binding analyses of $\mathrm{O} 1 \mathrm{~s}, \mathrm{C} 1 \mathrm{~s}$ and $\mathrm{N} 1 \mathrm{~s}$ peaks were carried out using synthetic line shapes consisting of a convolution product of a Gaussian function with $25 \%$ of a Lorentzian function for the different components. AES analysis was performed using a field emission gun operating at $10 \mathrm{kV}$ accelerating voltage, with a $3 \mathrm{nA}$ beam current resulting in a $150 \mathrm{~nm}$ spot size. AES spectra were recorded in the range of $50-600 \mathrm{eV}$ with a step size of $1 \mathrm{eV}$ and the analyzer operating in fixed retard ratio mode with a constant retard ratio of 2.

\subsubsection{Atomic force microscopy.}

Atomic force microscopy (AFM) analysis was carried out on a Nanowizard III (JPK Instruments AG, Germany). Experiments were performed using the Quantitative Imaging® force curve based imaging mode. In this mode, lateral forces applied by the AFM tip are minimized by a precise and continuous control of vertical forces during the approach and retract steps, thus avoiding pushing away or moving around the nanoparticles. Extend and retract curves were prepared at a constant speed of $50 \mu \mathrm{m} \mathrm{s}^{-1}$ upon a total extension of $100 \mathrm{~nm}$. The images were obtained from a $500 \mathrm{~nm}^{2}$ surface, scanning with 256 by 256 pixels. A standard AFM cantilever with a stiffness value of $0.35 \mathrm{~N} \mathrm{~m}^{-1}$ measured by thermal noise, and a tip apex of about $30 \mathrm{~nm}$ was used. While imaging, the maximum applied force was in the range of 8-15 $\mathrm{nN}$. The presented AFM images were only subjected to the first order plane flattening posttreatment.

\section{Results and discussion}

\subsection{Properties of $B W F$}

After filtration, BWF became less opaque and no sedimentation of particles was observed for several months. Notably, a peak between 200 and $220 \mathrm{~nm}$ on its UV spectrum indicated the presence of lipids, but since many molecules absorb in this area, it might also be due to aromatic pollutants. A broad shoulder between 230 and $300 \mathrm{~nm}$ was probably due to protein and nucleic acid absorption. Other molecules expected to be found in BWF are mucins, heme and nicotine, which also absorb and/or scatter UV light. DLS data showed that the filtration successfully removed particles larger than $350 \mathrm{~nm}$ (hydrodynamic diameter). The remaining population had a dispersity index of 0.169 and apart from a narrow peak at around $40 \mathrm{~nm}$, most particles were 
distributed between 100 and $350 \mathrm{~nm}$. The total amount of protein in BWF was $42.67 \mu \mathrm{g} \mathrm{ml}^{-1}$, however, the chromogenic effect of the assay depends on the basic residues of proteins, and the chlorination of $\alpha$-amino groups by $\mathrm{OCl}-$ could lead to the underestimation of the real amount of protein molecules in BWF.26 In any case, its strong UV extinction spectrum in combination with its ability to scatter light and an elevated relative density (1.094) point to a considerable load of small particles of an endogenous or exogenous origin. The biochemical and physicochemical data on BWF are presented in Fig. 3.

$\mathrm{NaOCl}$ is a common reagent added to pulmonary lavages prior to mineralogical analyses.7 Once dissociated, $\mathrm{OCl}-$ and $\mathrm{HOCl}$ can disrupt lipidic membranes and cleave macromolecules. A small amount of $\mathrm{NaOCl}$ was used in this study to disinfect the samples and prevent bacterial growth. Because of its destructive effect, cellular populations harvested by BAL might be partially destroyed, a caveat that should be taken into consideration when trying to distinguish nanoparticles trapped in the pulmonary hypophase from those internalized by cells (e.g., phagocytosed by macrophages), and those deposited on the pulmonary epithelium. In these cases, it is suggested that BAL fluids are treated soon after they have been harvested so that intact cellular structures can be isolated. Having said that, insoluble particles that persist in oxidative environments are often the culprits for chronic pulmonary inflammation and $\mathrm{NaOCl}-$ treated samples may be stored and retrospectively analysed in large, monocentric epidemiological studies. The effect of $\mathrm{NaOCl}$ on other types of nanoparticles is presented in Supplementary Information Table S3.

\subsection{Characterization of stock $A u N P s$}

The violet color of the stock AuNP dispersion was due to the surface plasmon resonance effect, typical of gold colloids of this size. Their hydrodynamic diameter in deionized water and their z-potential in PBS were $74.8 \mathrm{~nm}$ and $-44.3 \mathrm{mV}$, respectively. Their average primary particle size as measured by TEM was $62 \mathrm{~nm}$ and their citrate coating extended $\approx 1.0 \mathrm{~nm}$ above their surface. FESEM and TEM images revealed a multifaceted morphology typical of gold nanoparticles. Both the dispersity index as measured by DLS (0.126) and the spread of their primary size, as observed by TEM $(62 \pm 8 \mathrm{~nm}, \mathrm{~N}=180)$, indicated a narrow size distribution, but not a monodisperse population. When added in BWF, their intensity-weighted DLS signal was masked by the overwhelming native signal intensity of BWF. The characterization data on stock AuNPs are presented in Fig. 4. Direct observation of AuNPs in BWF by electron microscopy was impossible, as shown in a later section. 


\subsection{Extraction of AuNPs from BWF}

Images of the sample spiked with $19.0 \times 10^{8}$ AuNPs provided easily observable optical cues and are presented in Fig. 5. After the centrifugation in Step I, a violet band appeared in the cushion (Fig. 5, left panel). This was an indication that AuNPs agglomerate in BWF and sediment in clusters: the band's large width indicated that they undergo a varying degree of agglomeration and their color suggested that their clustering is loose enough to still allow for the plasmon resonance effect to manifest. The attempt to harvest them at the end of Step I would be ill-advised, given that the band was not sharp enough. Tubes were punctured at $\mathrm{d}_{1}=3 \mathrm{~mm}$ beneath the BWF-cushion interface and the supernatant volume was transferred to the next step.

After the centrifugation in Step II, a dark-colored speck was visible at the bottom of the tube (Fig. 5, right panel). The pelleting of AuNPs verified their agglomeration given that it would theoretically require a longer duration of time for monodisperse gold particles to travel the entire cushion. At this point, cup-horn sonication was performed to prevent pelleted AuNPs from adhering to the tube's wall. Sonication parameters (duration, amplitude, and the volume of water in the cup) were chosen empirically so as not to create a turbulent flow in the samples and disturb the glycerol gradient. The extent of interdiffusion as calculated by using eqn (5) was in good agreement with the macroscopic observation of the tubes were facilitated by the stark color difference between the aliquot and cushion. Finally, sBWAu was collected by aspiration after puncturing the tubes at $\mathrm{d}_{2}=2.1 \mathrm{~mm}$ beneath the initial aliquot-cushion interface; pBWAu was reconstituted to $1.00 \mathrm{ml}$ with the addition of $0.1 \mathrm{mM} \mathrm{NaOH}$ and the pellet was readily dispersed after a few seconds of cup-horn sonication.

\subsection{Characterization of the glycerol cushion}

The amount of glycerol in a liquid sample affects the implementation of bulk characterization techniques sensitive to fluctuations in viscosity, like DLS and ICP-OES. Glycerol in aqueous solutions can be accurately and precisely quantified by measuring their TOC. At the end of Step II (240 min), the glycerol content of pBWAu was $0.0037 \mathrm{~mol}( \pm 0.0006)$ which corresponds to $116 \%$ of its nominal value according to eqn (2) and (6). The total amount of glycerol in the sample was found to be $0.0069 \mathrm{~mol}( \pm 0.0006)$, which accounted for $95 \%$ of the theoretically calculated value $(0.0072 \mathrm{~mol})$. The above deviations can be attributed to imprecisions in the modeling of glycerol diffusion and the manual handling of the tubes. Importantly, the distribution of glycerol to sBWAu and pBWAu did not vary along the three time points which 
verified that the extent of interdiffusion was not underestimated. TOC results are summarized in Fig. 6A and B.

The cushion where AuNPs were extracted was not expected to be contaminated with biological species because its relative density was higher than most subcellular organelles and the applied centrifugal forces were not strong enough to sediment soluble molecules, like proteins or lipids. Indeed, the comparison of the FTIR spectra of glycerol cushions from blank BWF and negative samples showed that there were no qualitative differences between them (Fig. 6C). Specifically, both spectra indicated the presence of a glycerol-water solution: - $\mathrm{OH}$ bond stretching created the band between 3700 and $3100 \mathrm{~cm}^{-1}$, bending of the hydrogen bonds of water molecules gave rise to the peak at $1625 \mathrm{~cm}-1$, and the two shoulders around $2900 \mathrm{~cm}^{-1}$ were typical of glycerol. Only at $\approx 530 \mathrm{~cm}^{-1}$ did the two spectra slightly differ with a shoulder peak in the negative sample pointing to a weak presence of aromatic rings. Similarly, the Pierce total protein assay did not detect any protein content, but it has to be noted that the assay has not been tested by the manufacturer at such high glycerol concentrations. Concluding, BWF residues might be present in $\mathrm{pBWAu}$ due to imperfect harvesting of the sBWAu fraction, albeit in minute amounts that did not interfere with the characterization of the extracted AuNPs, as shown in section 3.5.

\subsection{Characterization of extracted AuNPs}

\subsubsection{Quantitative assessment.}

The Au content of pBWAu fractions was measured by ICP-OES: their Au signal intensities were compared against a matrix-matched calibration curve prepared with known amounts of AuNPs (Fig. 7A). A stable dispersion is necessary for measuring undigested metal particles with this technique. DPBS was found to be a good dispersant for AuNPs in glycerol-rich samples as DLS measurements showed that they did not sediment for nearly $3 \mathrm{~h}$ after their preparation at a concentration of $2.4 \times 10^{8}$ particles per ml (Fig. 7B). BWF samples were spiked with AuNPs at concentrations that ranged from $2.4 \times 10^{8}$ and $19.0 \times 10^{8}$ particles per ml. Given that fine particle concentration in human pulmonary lavages has been shown to range between $10^{5}$ and $10^{9}$ particles per $\mathrm{ml},{ }^{27,28}$ ultrafine particles are expected to be present at even higher values. The AuNP extraction yield ranged from 61 to $86 \%$ (Fig. 7B). The best yield was achieved with the least concentrated sample while the poorest was observed for the most concentrated sample, suggesting that agglomerated particles may be inadvertently excluded during Step I. Inversely, to rule out the possibility that only clustered AuNPs were successfully extracted, we performed the same method on undiluted fetal bovine serum where citratecapped AuNPs have exhibited low agglomeration29 (Fig. S4 of the Supplementary 
Information). Importantly, the relative standard deviation (RSD) of all recorded signal intensities was less than $6 \%$. The method's limit of detection for dissolved Au was calculated to be $18 \mu \mathrm{g} \mathrm{L}^{-1}$, whereas the lowest concentration of $\mathrm{Au}$ in particulate form that we attempted to detect amounted to $0.5 \mu \mathrm{g} \mathrm{ml}^{-1}$, hence this would be the method's limit of quantitation.

\subsubsection{Hydrodynamic size and dispersity.}

In DLS, the amount of light scattered by the observed volume of liquid generates a photon count per second. Because the power of the incident laser beam may be attenuated (so that the detector receives the optimum number of photons), the photon count rate is normalized to what it would have been, had the laser beam shined at its full power. Its normalized value is called "derived kilo-count rate per second" ( $\mathrm{dkcps}$ ) and is useful for comparing the intensity of light scattered by samples with differing particle contents. ${ }^{30,31}$ The dkcps signals of all pBWAu fractions were significantly stronger than that received from the negative sample and became progressively stronger than BWF spiked with increasing concentrations of AuNPs (Fig. 8A).

For the most part, pBWAu correlograms appeared to decay around the same time-point, indicating comparably-sized particle populations for all samples; they also exhibited similar gradients, suggesting comparably polydisperse particle populations (Fig. 8B). On the contrary, correlograms clearly differentiated at longer delay times, with lower concentration samples exhibiting stronger agglomeration. Furthermore, the point where the correlation curve intercepts the y-axis tended to smaller values for pBWAu fractions at higher particle concentrations, indicating a poorer signal-to-noise ratio. The reason for this decrease could be high glycerol concentration: increased viscosity allows for slowly fluctuating light intensities from residual biomolecules, thus generating a signal that is accounted for by the instrument. ${ }^{32,33}$ Because glycerol scatters light by itself - and in combination with increased AuNP concentration - lower intercept values could signify the onset of multiple scattering effects. ${ }^{34}$ Intercept values were above 0.8 , showing that the obtained data were of good quality and could be used to extrapolate the hydrodynamic size distribution of dispersed particles.

The dkcps-weighted peaks were multimodal: AuNPs were organized mainly in single particles or in few-particle clusters (Fig. 8C). To a much smaller extent, there were agglomerates of several hundred nanometers. According to Rayleigh scattering theory, objects smaller than the wavelength of the incident light beam scatter light at an intensity proportional to the sixth power of their diameter. ${ }^{35}$ Similar behavior was observed from samples containing clusters with hydrodynamic diameters larger than $633 \mathrm{~nm}$, but it has been theorized that Rayleigh scattering may still be exhibited by loosely agglomerated nanoparticles. ${ }^{36}$ In all but one pBWAu fraction, 
most of the scattered light (>94\%) came from the agglomerates of hydrodynamic diameters less than $500 \mathrm{~nm}$. The least concentrated sample was the sole exception with $16 \%$ of its scattered light coming from micron-sized, agglomerated AuNPs. Agglomeration of nanoparticles in the presence of biomolecules has been investigated by others and is known to be a dynamic process that depends on the nature and concentration of biomolecules, the size and surface properties of the particles, and the physicochemical properties of the continuous phase. ${ }^{37,38}$ Here, high glycerol concentration is expected to stabilize protein-covered AuNPs as well as denatured, aggregated protein clusters, hence the peak between 25 and $50 \mathrm{~nm}$ at lower particle concentrations. According to the manufacturer, the instrument is unable to resolve particle populations of which the hydrodynamic size differs by a factor $<1.7^{39}$ and since AuNPs are stronger scatterers than biomolecules, this small peak was merged with the peak from AuNPs, as their concentration increased (Fig. 8C).

At this point, it has to be clarified that the measured hydrodynamic size of AuNPs is not expected to be representative of their state in the lungs. In fact, as soon as BAL is performed, any retrieved particles are dispersed in a medium (saline) with a different composition than the pulmonary hypophase, therefore their organization will most likely adapt to their new environment. Also, there are other metrology techniques for small particulate matter in liquid dispersions that merit mentioning. For example, nanoparticle tracking analysis (NTA) is a rapidly emerging alternative to DLS that under specific conditions may distinguish dispersed particles with different refractive indices and resolve similarly-sized particle populations. ${ }^{40}$ Also, new fabrication techniques have enabled the engineering of nanosized pores on microfluidic sensors for the resistive pulse sensing of particles: even within multimodal populations, they present an excellent ability to resolve the absolute concentration and individual sizes of particles, while the recorded events can be used to extrapolate their shape, charge, and conductivity. ${ }^{41}$

\subsubsection{Size and nanoscale characteristics.}

BSE analysis of the most concentrated pBWAu sample showed that AuNPs were observable at low magnification due to the high atomic number of gold and the removal of biological noise which can otherwise smother objects that do not protrude above the level where the electron beam is focused (Fig. 9A). Under conventional FESEM, the substrate presented minimal artefacts, so the particle morphology, agglomeration state, and their partial covering by organic residues were easily discernible (Fig. 9B). Under higher magnification, there were signs of bridging between particles, probably due to their biomolecular coating acquired in BWF (Fig. 
9C). For comparison purposes, FESEM and BSE images of an untreated BWF sample spiked with $19.0 \times 10^{8}$ AuNPs are presented in Fig. 9D. It is evident that the biological material smothers the signal and prevents their morphological analysis. The BSE detector revealed some particles hidden in the biological material, but those lying deeper could not be resolved, hence the muddled image (Fig. 9E). The extracted AuNPs could be observed under high resolution TEM, too: the grid appeared mostly free from artefacts and the particles' Feret diameter was easily observable (Fig. 9F-H). Moreover, their coating appeared thicker than their original citrate layer (Fig. 9I), but it could not be measured due to the lack of staining agent. Given the changes in their physicochemical environment during the extraction procedure and washing cycles, this coating is expected to be composed of more firmly adhered biomolecules, referred to as a "hard biomolecular corona".

To demonstrate its clinical utility, the particle extraction method was applied on bronchial washing fluids from 2 patients. DLS, ICP-OES, and electron microscopy data on particles extracted from these samples are presented in File S5 of the Supplementary Information.

\subsubsection{Surface chemistry and morphology.}

XPS analyses allowed for the identification of elements and chemical bonds at the surface of the stock and extracted AuNPs using high resolution energy spectra. Moreover, the thickness of the layer covering the extracted AuNPs was estimated using the Shard method for interpreting XPS data from core-shell nanoparticles. ${ }^{42,43}$ Given the $400 \mu \mathrm{m} 2$ spot-size of the focused X-ray beam, XPS analyses give averaged information on a population of nanoparticles. However, subsequent AES analyses with a lateral resolution of $\approx 150 \mathrm{~nm}^{2}$ allowed for the identification of elements like sulphur $(\mathrm{S})$ and nitrogen $(\mathrm{N})$ on the surface of few agglomerated AuNPs following their extraction from BW, which are good indicators of the presence of proteins. Finally the surface morphology of the stock and extracted AuNPs was compared using AFM analyses. XPS survey scans acquired by the stock and extracted AuNPs are presented in Fig. 10. The two spectra exhibit common elements: gold $(\mathrm{Au})$ from the nanoparticles, silicon (Si) from the wafer on which the nanoparticles were deposited, as well as oxygen $(\mathrm{O})$, carbon (C), sodium $(\mathrm{Na})$ and chlorine $(\mathrm{Cl})$. The $\mathrm{N}$ 1s photoelectron peak, indicative of the presence of $\mathrm{N}$, was observed only for the specimen with extracted AuNPs, suggesting the existence of proteins on their surface. Moreover, it can be noticed that $\mathrm{Au} 4 \mathrm{~d}$ and $\mathrm{Au} 4 \mathrm{f}$ peaks are more intense for stock AuNPs. In particular, the ratios of the intensity of $\mathrm{Au} 4 \mathrm{f}$ to the intensity of $\mathrm{Si}$ $2 \mathrm{p}$ are respectively 0.114 and 2.87 for the sample with the stock AuNPs and the one with the extracted AuNPs. The intensities of photoelectron peaks associated with Au are dependent on 
the coverage of particles on the analyzed area of the silicon wafer as well as on the attenuation due to an overlayer at the surface of nanoparticles. Given that the analyzed area of the silicon wafer was similarly covered by both types of particles, it is reasonable to assume that the organic overlayer is thicker in the case of extracted AuNPs, an observation coherent with the presence of a biomolecular corona.

High-energy XPS resolution scans of C 1s, O 1s, and N 1s peaks for the stock and extracted AuNPs as well as a core-shell representation of these nanoparticles with organic shell thicknesses calculated according to the Shard method can be seen in Fig. 11. As expected, the shapes of the $\mathrm{C} 1 \mathrm{~s}$ and $\mathrm{O} 1 \mathrm{~s}$ photoelectron peaks resulting from the different oxygen and carbon chemical bindings are very different between the stock and extracted AuNPs. For the first ones, the overlayer consists of citrate molecules hence the $\mathrm{C} 1 \mathrm{~s}$ peak (Fig. 11A) can be deconvoluted using four components, as described in the work of Park and Shumaker-Parry:44 the adventitious carbon on the silicon wafer $(\mathrm{C}-\mathrm{C}, \mathrm{C}-\mathrm{H})$ at $284.8 \mathrm{eV}$, the hydroxyl $(\mathrm{C}-\mathrm{OH})$ and/ or the $\alpha$-carbons $\left(\mathrm{CH}_{2}\right)$ at $285.9 \mathrm{eV}$, the coordinated carboxylates $(\mathrm{COO}-\mathrm{Au})$ at $287.6 \mathrm{eV}$ and the free carboxyl moieties (COOH or COO-) at $289 \mathrm{eV}$. For O 1s (Fig. 11C), the contributions were from the $\mathrm{Si}-\mathrm{O}$ binding due to the silicon wafer native oxide and from oxygen present in the citrate layer $(\mathrm{C}-\mathrm{O}$ and $\mathrm{C}=\mathrm{O})$. Concerning the extracted AuNPs, the core level spectra of $\mathrm{C}$ 1s (Fig. 11D) were deconvoluted using components that were assigned to proteins and/or lipids present at the surface of those particles, as demonstrated in the work of Das et al. ${ }^{45} \mathrm{C}-\mathrm{C}$ and/or $\mathrm{C}-\mathrm{H}$ at $284.8 \mathrm{eV}$ (part of this component may originate from the adventitious carbon), $\mathrm{C}-\mathrm{N}$ at $286.4 \mathrm{eV}$ for amine groups, $\mathrm{O}-\mathrm{C}-\mathrm{O}$ and/or $\mathrm{C}-\mathrm{OH}$ at $287.6 \mathrm{eV}$ for ether and alcohol groups, and, finally, $\mathrm{O}=\mathrm{C}-\mathrm{OH}$ and $\mathrm{N}-\mathrm{C}=\mathrm{O}$ at $289.4 \mathrm{eV}$ which take into account possible carboxylate and amide carbon groups in protein molecules. For the $\mathrm{O} 1 \mathrm{~s}$ core level (Fig. 11F), in addition to the $\mathrm{Si}-\mathrm{O}$ component for the silicon wafer native oxide, a contribution from the amide group $\mathrm{O}=\mathrm{C}-\mathrm{N}$ was found at $536 \mathrm{eV}$ as well as a $\mathrm{C}-\mathrm{O}$ and/or $\mathrm{C}=\mathrm{O}$ component at $532.7 \mathrm{eV}$. Finally, for the $\mathrm{N}$ 1s (Fig. 11E) spectra, three components at 399.5, 401.7 and $403 \mathrm{eV}$ were assigned to amide, unprotonated and protonated amine groups present in protein molecules, respectively. The organic shell thickness of the stock AuNPs was calculated based on the Shard method to be $\approx 1 \mathrm{~nm}$ with carbon and oxygen atomic fractions of 0.47 and 0.53 which is coherent with the presence of citrate molecule capping. For extracted AuNPs, the same method gives an organic shell thickness of $\approx 6 \mathrm{~nm}$, itself coherent with the presence of biomolecules. The atomic fractions of $\mathrm{C}, \mathrm{O}$ and $\mathrm{N}$ in the shell were estimated to respectively $0.75,0.19$ and 0.06 . The results of the calculations of the organic shell thickness and composition on the stock and extracted gold 
nanoparticles are summarized in the schematic of Au nanoparticles in Fig. 11. The details of these calculations based on the intensities ratios of signals coming from the core $(\mathrm{Au} 4 \mathrm{f})$ and signals coming from the organic shell ( $\mathrm{C} \mathrm{1s,} O \mathrm{O}$ 1s and $\mathrm{N}$ 1s) are presented in File S6 of the Supplementary Information. AES analyses were performed on both types of Au nanoparticles in order to take advantage of the higher lateral resolution $\left(\approx 150 \mathrm{~nm}^{2}\right)$ of this method. For each sample, analyses were carried out on ten different areas. Each area corresponds to one or few agglomerated nanoparticles. Additional analyses were performed on the substrate near the extracted AuNPs in order to exclude the possibility of unassociated biomolecules contributing to the obtained signals. Finally, as described in the Materials and methods part, the wafer substrates were coated with a thin palladium film before the deposition of AuNPs in order to avoid any charging effects. Examples of the AES spectra acquired on the substrate, stock and extracted AuNPs are presented in Fig. 12A. Auger transitions $S_{L V V}, N_{K L L}$ which are expected to come from the $\mathrm{N}$ content of proteins were only detected in the case of extracted AuNPs. Moreover, the transition $\mathrm{Au}_{\mathrm{NVV}}$ is less intense for the extracted nanoparticles than for the stock ones which corroborates the difference in the thickness of the organic shell previously detected by XPS analyses. The transitions $\mathrm{C}_{\mathrm{KLL}}$ and $\mathrm{O}_{\mathrm{KLL}}$ appear on the spectrum for the stock AuNPs due to the citrate layer, but they are less pronounced than for the extracted AuNPs. Finally, the $\mathrm{S}_{\mathrm{LVV}}$ and $\mathrm{N}_{\mathrm{KLL}}$ transitions were not found in the spectrum of the substrate in areas near the extracted AuNPs, which again indicates that biomolecules are adsorbed on the nanoparticles and not dispersed on the wafer substrate.

Regarding the biochemical composition of the organic shell, it needs to be clarified that its structural integrity may not be preserved after the addition of $\mathrm{NaOCl}$. Chromatographic, spectrometric, or electrophoretic techniques need to verify that the primary protein structure is not heavily oxidized and adsorbed proteins are still identifiable.

AFM analysis on a single stock AuNP is presented in Fig. 12B where the particle's characteristic angular shape is visible; in Fig. 12B another particle appears covered by an irregular shell. Their cross-sectional profiles can be seen in Fig. 12D: the difference between the width and height is due to the convolution of the recorded topographic signals with the apex of the AFM tip $(\approx 25 \mathrm{~nm})$. However, the difference in the tails of the cross-sectional profiles for the two nanoparticles suggests the presence of an organic shell on the particle extracted from BW. Moreover the difference in the maximum height $(<10 \mathrm{~nm})$ is in good agreement with the thickness of the organic shell for extracted AuNPs calculated previously by XPS analyses. 


\section{Conclusions}

In the present study, we show that the thermodynamically unstable interface between glycerol solutions and human bronchoalveolar lavage fluids can be exploited for the quantitative extraction of sub- $\mu \mathrm{g} \mathrm{ml}^{-1}$ AuNPs under relatively low centrifugal forces. The time required for particle extraction using common lab equipment was calculated by modeling their movement according to fundamental principles of particle sedimentation and liquid-liquid interdiffusion. The limit of quantitation for elemental Au at $0.5 \mu \mathrm{g} \mathrm{ml}^{-1}$ corresponds to $2.4 \times 10^{8}$ particles per ml. Eventually, AuNPs were extracted individually and in loose clusters but, at higher concentrations, particle agglomeration may downgrade the method's efficiency. The hydrodynamic size distribution of extracted AuNPs was resolved by DLS, although it is not expected to be representative of their organization on the pulmonary surface. Also, the recorded photon count rates significantly depended on particle concentration, suggesting that the combination of DLS and ICP-OES data could be indicative of relatively elevated, metalcontaining, submicron and nanosized particle burdens in human bronchial washing fluids.

Due to the achieved extraction yields and the successful segregation of soluble biological noise, extracted AuNPs could be observed at the nanoscale: BSE imaging was used to detect the dense gold particle cores at low magnification, and TEM and FESEM could be used to resolve the particles' Feret diameter and morphology, respectively. Finally, the preserved biomolecular corona of the extracted AuNPs was studied by AFM, AES, and XPS which provided information on its morphology, elemental composition, and chemistry, respectively. Overall, we believe that this work provides a solid method for the quantitative and qualitative analysis of gold nanoparticles in human bronchoalveolar lavages. The detailed description and theoretical modeling of the protocol renders it potentially useful for the exploration of the nanoparticle load of human biological samples and could help transition clinical nanotoxicology from case studies to more systematic investigations. Still, it is important to note that any new iteration of the procedure with different biological samples and/or different types of nanoparticles has to be verified with standalone, additional experiments.

\section{Study approval and consent to participate}

Bronchoalveolar lavage fluids and clinical data were retrieved from patients admitted to the University Hospital in Saint-Etienne in the context of a clinical study approved by the French National Agency for Drug and Health Products Safety (Agence Nationale de Securité du Médicament et des Produits de Santé) with the reference number B121211-32 and the protocol 
number 1008122 ("NanoPi”). Patients were informed and gave their full written consent prior to their participation in the study.

\section{Author contributions}

DiB designed the extraction method, performed analytical experiments and wrote the manuscript; VB performed surface analyses and wrote part of the manuscript; JMV, CG collected bronchoalveolar lavages and clinical data; PC provided knowhow in Matlab scripting; DeB, VF, JP, JMV, and MC evaluated data and commented on the manuscript; JMV conceived the clinical study. All authors read and approved the final manuscript.

\section{Conflicts of interest}

All authors have read and approved the manuscript for publication and declare that they have no competing interests. Raw analytical data and the Matlab script are made available by the authors upon request.

\section{Acknowledgements}

We appreciate Alexandre Govin and Marie-Claude Bartholin for their help with TOC experiments; Sergio Sao-Joao and Marilyne Mondon for their help with transmission electron microscopy; Laura Morel and Jacques Moutte for the realization of ICP-OES measurements. DiB would also like to thank Anastasios Ragkousis for his constructive comments. This work has been partially funded by the Funds for Research in Respiratory Health (Fonds de Recherche en Santé Respiratoire).

\section{References}

1 NSTC, Subcommittee on Nanoscale Science, Engineering and Technology. Progress Review on the Coordinated Implementations of the National Nanotechnology Initiative. Environmental, Health, and Safety Research Strategy, online, 2014, http://www.nano.gov/sites/default/files/pub_resource/2014_nni_ehs_progress_review.pdf.

2 G. Roebben, V. Kestens, Z. Varga, J. Charoud-Got, Y. Ramaye, C. Gollwitzer, D. Bartczak, D. Geißler, J. Noble, S. Mazoua, et al., Front. Chem., 2015, 3, 1-16.

3 D. Pelclova, H. Barosova, J. Kukutschova, V. Zdimal, T. Navratil, Z. Fenclova, S. Vlckova, J. Schwarz, N. Zikova, P. Kacer, et al., J. Breath Res., 2015, 9, 036008.

4 Y. Song, X. Li and X. Du, Eur. Respir. J., 2009, 34, 559-567. 
5 D. Bitounis, J. Pourchez, V. Forest, D. Boudard, M. Cottier and J.-P. Klein, Biomaterials, 2016, 76, 302-312.

6 R. Goldstein, P. Rohatgi, E. Bergofsky, E. Block, R. Daniele, D. Dantzker, G. Davis, G. Hunninghake, T. King Jr. and W. Metzger, Am. Rev. Respir. Dis., 1990, 142, 481-486.

7 P. De Vuyst, A. Karjalainen, P. Dumortier, J. C. Pairon, E. Monso, P. Brochard, H. Teschler, A. Tossavainen and A. Gibbs, Eur. Respir. J., 1998, 11, 1416-1426.

8 L. Paoletti, Arch. Environ. Health, 1997, 52, 384-389.

9 N. Kulkarni, N. Pierse, L. Rushton and J. Grigg, N. Engl. J. Med., 2006, 355, 21-30.

10 A. Karjalainen, R. Piipari, T. Mantyla, M. Monkkonen, M. Nurminen, P. Tukiainen, E. Vanhala and S. Anttila, Eur. Respir. J., 1996, 9, 1000-1005.

11 J. Kolosnjaj-Tabi, J. Just, K. B. Hartman, Y. Laoudi, S. Boudjemaa, D. Alloyeau, H. Szwarc, L. J. Wilson and F. Moussa, EBioMedicine, 2015, 2, 1697-1704.

12 M. A. Livshits, E. Khomyakova, E. G. Evtushenko, V. N. Lazarev, N. A. Kulemin, S. E. Semina, E. V. Generozov and V. M. Govorun, Sci. Rep., 2015, 5, 17319.

13 M. Witzler, F. Küllmer and K. Günther, Analytical Letters, 2017.

14 A. Tsuda and P. Gehr, Nanoparticles in the lung: environmental exposure and drug delivery, CRC Press, 2015.

15 R. A. Yokel and R. C. MacPhail, J. Occup. Med. Toxicol., 2011, 6, 7.

16 I. Du Rand, J. Blaikley, R. Booton, N. Chaudhuri, V. Gupta, S. Khalid, S. Mandal, J. Martin, J. Mills, N. Navani, et al., Thorax, 2013, 68, i1-i44.

17 W. Mächtle and L. Börger, Analytical ultracentrifugation of polymers and nanoparticles, Springer Science \& Business Media, 2006.

18 J. Crank, The mathematics of diffusion, Oxford university press, 1979.

19 R. W. Balluffi, S. Allen and W. C. Carter, Kinetics of materials, John Wiley \& Sons, 2005. 20 W. M. Haynes, CRC handbook of chemistry and physics, CRC press, 2014.

21 N.-S. Cheng, Ind. Eng. Chem. Res., 2008, 47, 3285-3288.

22 P. Haslam and R. Baughman, Eur. Respir. J., 1999, 14, 245-248.

23 H. E. Pace, N. J. Rogers, C. Jarolimek, V. A. Coleman, C. P. Higgins and J. F. Ranville, Anal. Chem., 2011, 83, 9361-9369.

24 M. H. Sousa, G. J. da Silva, J. Depeyrot, F. A. Tourinho and L. F. Zara, Microchem. J., 2011, 97, 182-187.

25 M. Moretti, D. Sisti, M. B. Rocchi and E. Delprete, Clin. Chim. Acta, 2011, 412, 11431145 . 
26 B. S. Antharavally, K. A. Mallia, P. Rangaraj, P. Haney and P. A. Bell, Anal. Biochem., 2009, 385, 342-345.

27 J.-C. Pairon, M.-A. Billon-Galland, Y. Iwatsubo, M. Bernstein, A. Gaudichet, J. Bignon and P. Brochard, Environ. Health Perspect., 1994, 102, 269.

28 C. Voisin, F. Fisekci, B. Buclez, A. Didier, B. Couste, F. Bastien, P. Brochard and J. Pairon, Eur. Respir. J., 1996, 9, 1874-1879.

29 E. Casals, T. Pfaller, A. Duschl, G. J. Oostingh and V. Puntes, ACS Nano, 2010, 4, 36233632 .

30 M. I. Ltd, Derived count rate - what is it?

31 P. Sun, D. Zhou and Z. Gan, J. Controlled Release, 2011, 155, 96-103.

32 B. Lorber, F. Fischer, M. Bailly, H. Roy and D. Kern, Biochem. Mol. Biol. Educ., 2012, 40, 372-382.

33 K. Elamin and J. Swenson, Phys. Rev. E: Stat. Phys., Plasmas, Fluids, Relat. Interdiscip. Top., 2015, 91, 032306.

34 T. Zheng, S. Bott and Q. Huo, ACS Appl.Mater. Interfaces, 2016, 8, 21585-21594.

35 J. Shang and X. Gao, Chem. Soc. Rev., 2014, 43, 7267-7278.

36 F. Babick, K. Schieß1 and M. Stintz, Part. Part. Syst. Charact., 2012, 29, 104-115.

37 A. A. Keller, H. Wang, D. Zhou, H. S. Lenihan, G. Cherr, B. J. Cardinale, R. Miller and Z. Ji, Environ. Sci. Technol., 2010, 44, 1962-1967.

38 J. M. Zook, R. I. MacCuspie, L. E. Locascio, M. D. Halter and J. T. Elliott, Nanotoxicology, $2011,5,517-530$.

39 M. I. Ltd, Application of Dynamic Light Scattering (DLS) to Protein Therapeutic Formulations: Principles, Measurements and Analysis.

40 Y. Yuana, R. M. Bertina, S. Osanto, et al., Thromb. Haemostasis, 2011, 105, 396.

41 J. Ko, E. Carpenter and D. Issadore, Analyst, 2016, 141, 450-460.

42 A. G. Shard, J. Phys. Chem. C, 2012, 116, 16806-16813.

43 N. A. Belsey, A. G. Shard and C. Minelli, Biointerphases, 2015, 10, 019012.

44 J.-W. Park and J. S. Shumaker-Parry, J. Am. Chem. Soc., 2014, 136, 1907-1921.

45 S. K. Das, J. Liang, M. Schmidt, F. Laffir and E. Marsili, ACS Nano, 2012, 6, 6165-6173. 


\section{Figure captions}

Figure 1 (A) Step I: Coarse particles advance into the cushion under centrifugal force rcf $_{1}$ over time $t_{1}$, while smaller particles are collected in the volume above the plane at distance $\mathrm{d}_{1}$ beneath the BWF-cushion interface. (B) Graphical representation of eqn (1) for set $\operatorname{rcf}_{1}$ and $\mathrm{t}_{1}$ values: the dashed curve corresponds to the smallest $\mathrm{d}_{1}$ value (nearest to the BWF-cushion interface). (C) Step II: The aliquot collected from the previous step is centrifuged under $\mathrm{rcf}_{2}$. The time $\mathrm{t}_{2}$ required for a particle to move to the part of the cushion that has not mixed with the aliquot is calculated by modeling its sedimentation. The extent of diffusion $\mathrm{d}_{2}$ over the said period of time defines the volume of the particle containing a fraction. (D) Graphical representation of eqn (3) and (4) for the spatial and temporal evolution of glycerol concentration at three time points (the black dashed curve corresponds to the shortest time point).

Figure 2 Under constant rotations per minute, the instantaneous velocity $U$ of a sedimenting AuNP depends on the medium's viscosity $(\eta)$ and density $(\rho)$ and on the particle's distance from the axis of rotation $(\mathrm{R})$. The dotted line at $\mathrm{x}=0.0 \mathrm{~mm}$ marks the initial interface between the aliquot and the cushion; the color scale on the top indicates the concentration of glycerol in mol L ${ }^{-1}$; the white dashed line traces the particle sedimentation route along the length of the tube, with the air-sample interface set as its starting point at $x=16.5 \mathrm{~mm}$. The white dots at $\mathrm{x}$ $\approx 5 \mathrm{~mm}$ and at $\mathrm{x}=0.0$ represent the particle as it moves through the aliquot and the glycerol gradient, respectively. The ordinate of the open black dot represents the minimum time required for the said particle to travel through the entire aliquot region and glycerol gradient; the modulus of the abscissa represents the distance from the interface at the said time point. Finally, the closed black dot represents the particle after it has gained a distance of $1 \mathrm{~mm}$ from the expanding glycerol gradient.

Figure 3 (A) Biochemical and physicochemical properties of BWF and blank BWF. (B) Extinction spectra of BWF at various dilutions in PBS.

Figure 4 (A) Properties of AuNPs in DPBS. (B) Intensity-weighted hydrodynamic size distribution of AuNPs in various media. (C, D) FESEM and TEM images of AuNPs, respectively. The white dashed rectangle is imaged under higher magnification in $(\mathrm{E})$ where a citrate layer of $\approx 1.0 \mathrm{~nm}$ was visible without staining. Scale-bars: C, D, $100 \mathrm{~nm} ; \mathrm{E}, 40 \mathrm{~nm}$. 
Figure 5 Step I: The tube was punctured at $\mathrm{d}_{1}=3.0 \mathrm{~mm}$ beneath the BWF-cushion interface and the collected aliquot was transferred to Step II (the excluded volume can be investigated for readily sedimenting, micron-sized particles). Step II: According to eqn (5), the extent of interdiffusion was $\mathrm{d}_{2} \approx 2.1 \mathrm{~mm}$, in good accordance with macroscopic observations. The biological sample and part of the glycerol cushion constitute the aspirated volume sBWAu. Extracted particles are contained in pBWAu where some have pelleted and can be seen in the magnified crop of the dashed rectangle.

Figure 6 (A) Schematic representation of aliquot-cushion interdiffusion (blue and yellow, respectively) at three time points (sizes not in the scale). (B) Mean glycerol values in sBWAu and pBWAu indicate that the puncture point for their separation lies outside the extent of interdiffusion. Differences between the theoretical and measured glycerol values may be due to imprecisions in puncturing the tubes. Error bars represent sample standard deviations (SD), with $\mathrm{n}=3$ for each time point per fraction. (C) Qualitative comparison of the FTIR spectra obtained from a cushion of blank BW and a negative sample.

Figure 7 (A) Calibration curve of AuNPs in a matrix with a similar composition to pBWAu: bands correspond to $99 \%$ confidence; linear regression had a fit of $r^{2}=0.998$. (B) AuNP extraction yields in pBWAu fractions from BW spiked at various particle concentrations: error bars represent sample SD with $n=3$. (C) Colloidal stability over 160 min of AuNPs added in a matrix with a similar composition to pBWAu: photon count rates and dispersity indices stay within narrow margins indicating good dispersibility.

Figure 8 (A) dkcps signals from pBWAu and their respective sBWAu fractions: ordinary oneway ANOVA test $(\mathrm{p}<0.0001)$ and post hoc multiple comparisons showed that the signals differed significantly between all pBWAu fractions $(\mathrm{n}=3)$; **p $<0.01$, ***p $<0.001$, ****p $<$ 0.0001. Their respective sBWAu signals show small variations $(\mathrm{RSD}=10.8 \%)$. Error bars represent sample SD (some are too small to be visible). (B) Averaged correlograms $(n=3)$ from pBWAu at various AuNP particle concentrations. (C) dkcps-weighted hydrodynamic size distributions of pBWAu fractions. (D) dkcps signals and the amount of AuNPs extracted from each BWF sample were strongly associated: Spearman's rank correlation coefficient $r_{s}=1$; *p $<0.05$. Error bars represent sample SD with $\mathrm{n}=3$ (too small to be visible on the $\mathrm{x}$ axis). 
Figure 9 (A) Extracted AuNPs imaged with BSE. The cluster in the white rectangle was observed with a conventional FESEM in-lens detector, as presented in (B) where black spots indicate carbon-rich materials of high work functions. (C) High magnification, in-lens imaging revealed the bridging of two particles (white arrow). (D) Direct imaging of AuNPs in BWF with the in-lens detector and with BSE as shown in (E), is problematic because particles are covered by the biological material. (F) Low magnification TEM images of extracted AuNPs: the cluster in the black rectangle was imaged at higher magnification and is presented in $(\mathrm{G})$. (H). High resolution TEM image of a cluster of AuNPs appears covered by a thicker layer than their original citrate coating; the area in the black rectangle is magnified in (I). Scale bars: A, 5 $\mu \mathrm{m} ; \mathrm{B}, 500 \mathrm{~nm}$; C, $100 \mathrm{~nm}$; D, E, $500 \mathrm{~nm}$; F, $1 \mu \mathrm{m}$; G, $100 \mathrm{~nm}$; H, $50 \mathrm{~nm}$; I, $10 \mathrm{~nm}$.

Figure 10 XPS survey scans of the samples of the silicon wafer with stock AuNPs (black curve) and extracted AuNPs (red curve).

Figure 11 Core/shell schematic illustration of a single gold nanoparticle with thickness and composition measurements of the organic shell as calculated for stock and extracted nanoparticles according to a method proposed by Shard. ${ }^{42}$ High resolution XPS scans of C $1 \mathrm{~s}$, $\mathrm{N} 1 \mathrm{~s}$ and $\mathrm{O} 1 \mathrm{~s}$ for stock AuNPs (A-C) and extracted AuNPs (D-F).

Figure 12 (A) AES spectra recorded from extracted (top) and stock AuNPs (middle), as well as from the wafer substrate covered with a Pd film near some extracted nanoparticles (bottom). The AFM picture of a stock Au nanoparticle (B) and of the one extracted from BW (C). (D) Cross-sectional profiles of the two nanoparticles. 


\section{Tables}

Table 1 Assumptions for the modeling of the extraction procedure

\section{Particles}

- particles are spherical with homogeneous density and monodisperse size distribution

- during sedimentation, particles do not collide with the tube walls

- there are no electrostatic interactions between particles and the tube

- particle diffusion due to Brownian motion is negligible compared to their sedimentation

- Reynold's number $<$ 0:2 (particle sedimentation does not cause turbulent flow)

\section{Pulmonary lavage fluids and glycerol solutions (liquids)}

- all liquids are isotropic

- viscosity and density depend only on their glycerol concentration

- interdiffusion is only driven by glycerol concentration gradient

- total volume of liquids stays constant

- liquid transfer is due to diffusion and there are no convective currents 


\section{Figure 1}

Step I

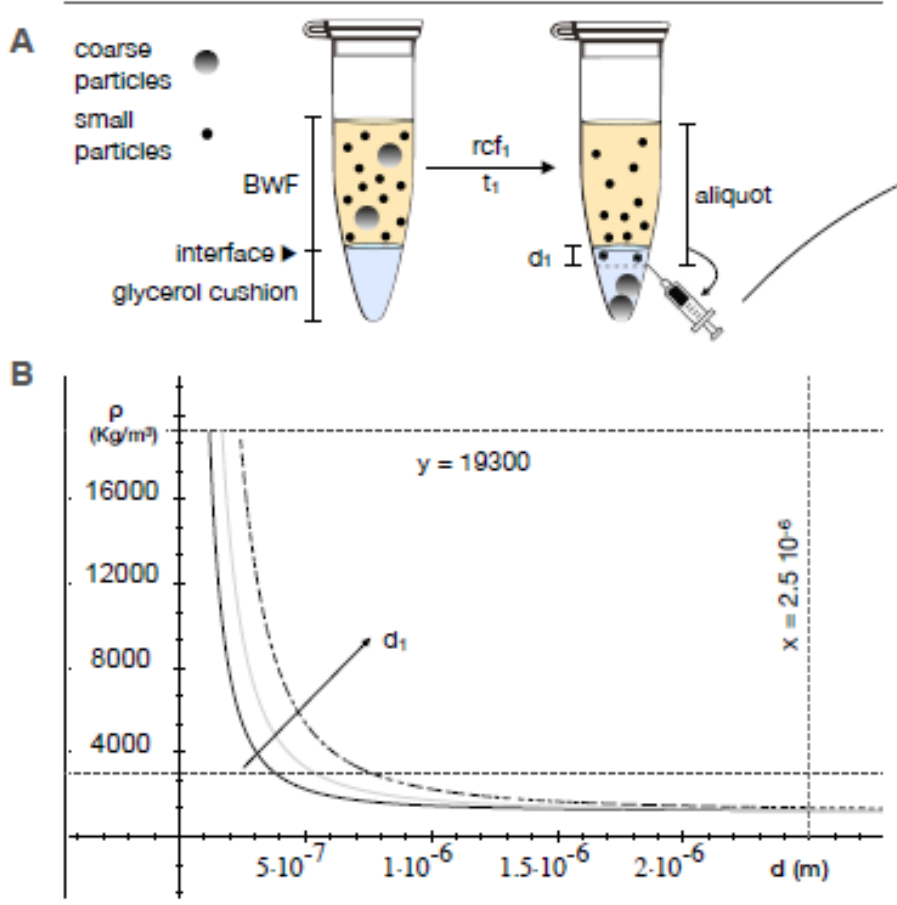

Step II

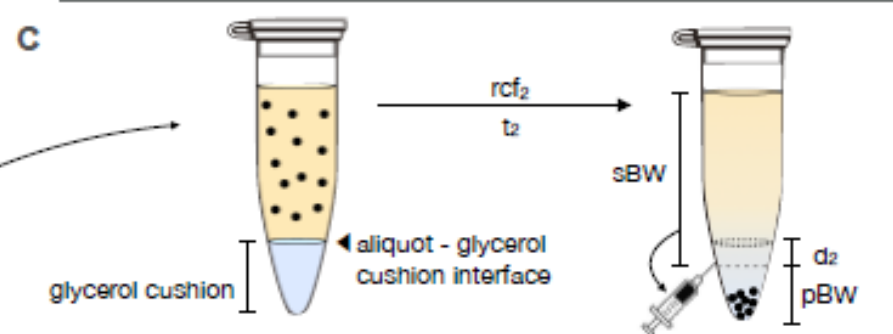

D



\section{Figure 2}
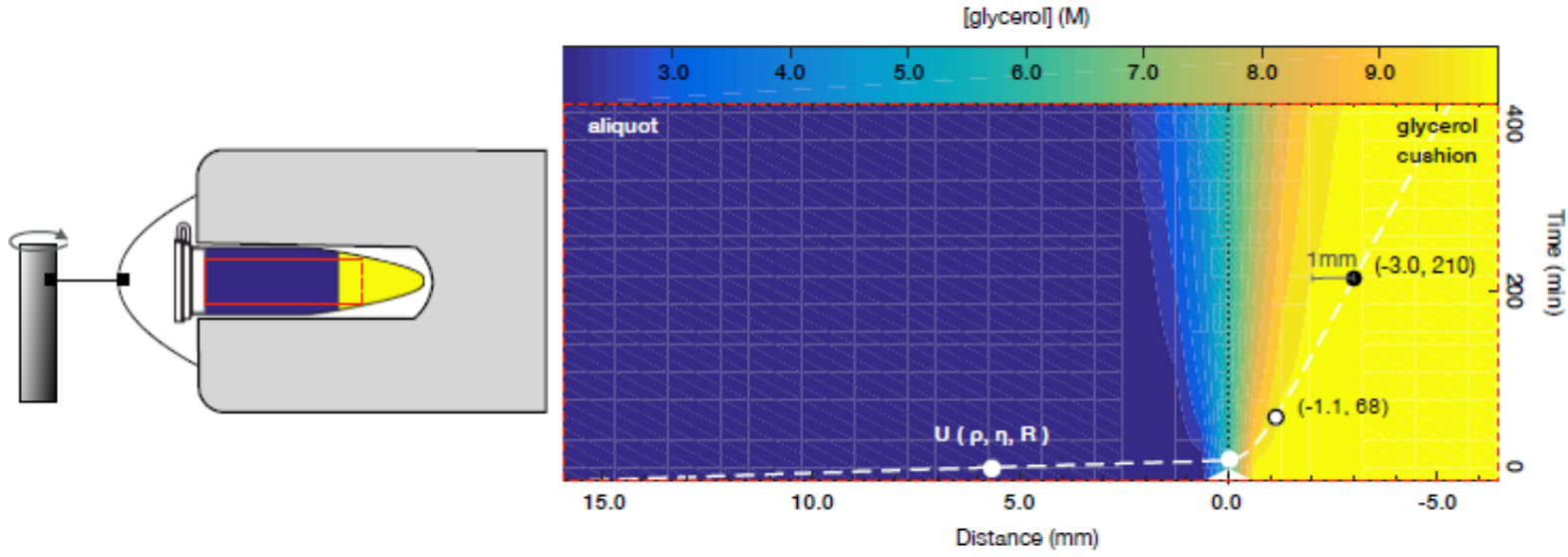


\section{Figure 3}

A

\begin{tabular}{|c|c|c|}
\hline \multirow{7}{*}{ BWF } & total protein $(\mu \mathrm{g} / \mathrm{ml})$ & $\begin{array}{l}42.67 \\
r^{2}=0.92\end{array}$ \\
\hline & relative deneity & 1.094 \\
\hline & S.D. & 0.000 \\
\hline & ocattered light (dkcpe) & $\overline{47680}$ \\
\hline & S.D. & 1942 \\
\hline & dispersity index & 0.169 \\
\hline & S.D. & 0.004 \\
\hline \multirow{4}{*}{$\begin{array}{l}\text { blank } \\
\text { BWF }\end{array}$} & $\mathrm{pH}$ & 11.35 \\
\hline & S.D. & 0.10 \\
\hline & ionic strength $(\mathrm{M})$ & 0.19 \\
\hline & reduction potential $(\mathrm{mV})$ & 0.87 \\
\hline
\end{tabular}

B

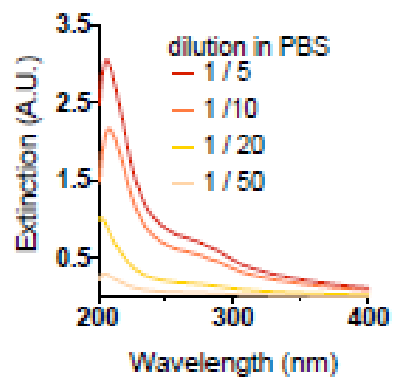

\section{Figure 4}

A

\begin{tabular}{l|l}
$\begin{array}{l}\text { primary size (nm) } \\
\text { S.D. }\end{array}$ & 62 \\
\hline scattered light (dkcps) & 14498 \\
\hline S.D. & 82 \\
\hline Z-Average (nm) & 74.8 \\
S.D. & 0.2 \\
\hline dispersity index & 0.126 \\
S.D. & 0.014 \\
\hline Z-potential (mV) & -44.3 \\
S.D. & 0.2
\end{tabular}

B

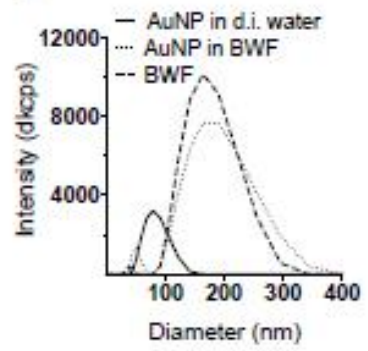



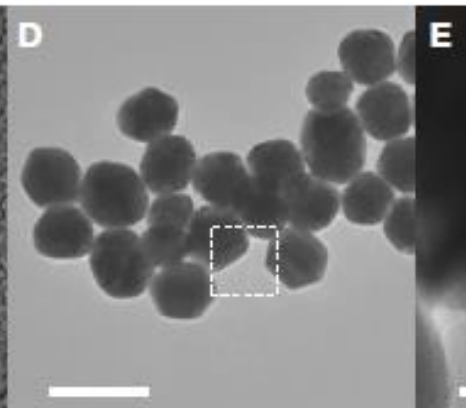

Figure 5

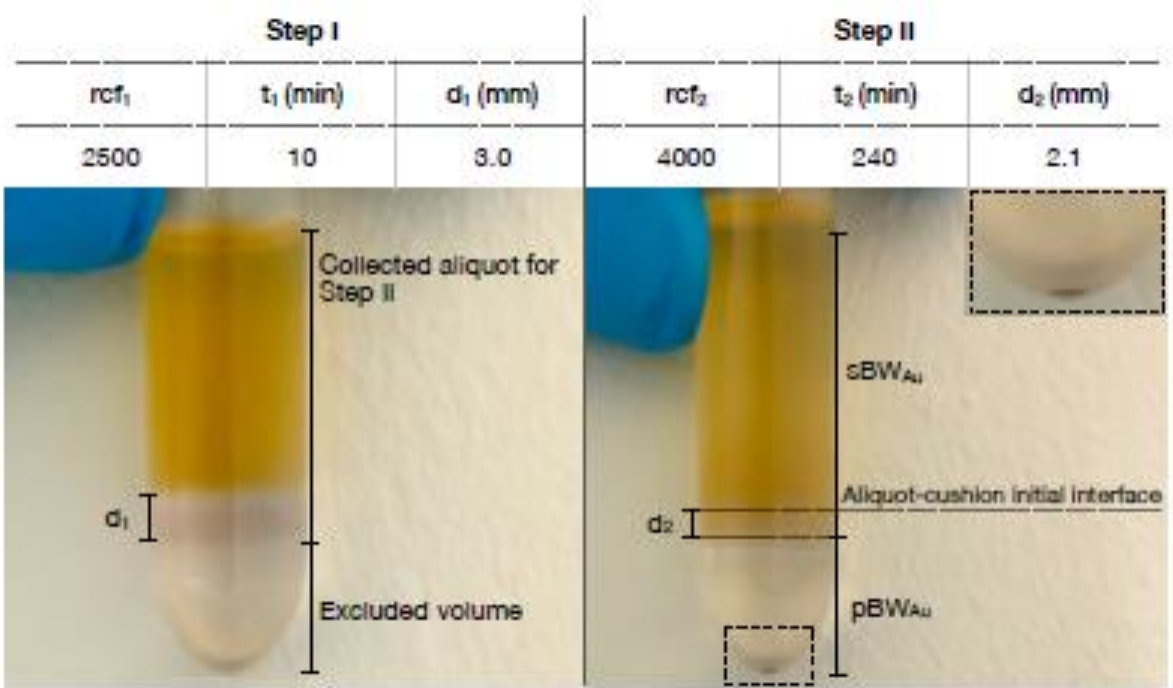


Figure 6

A

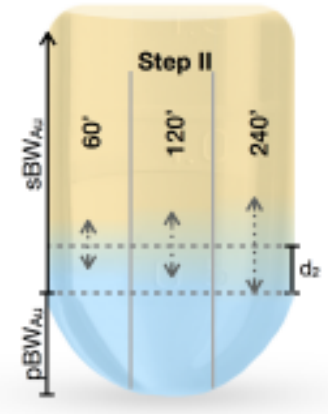

B

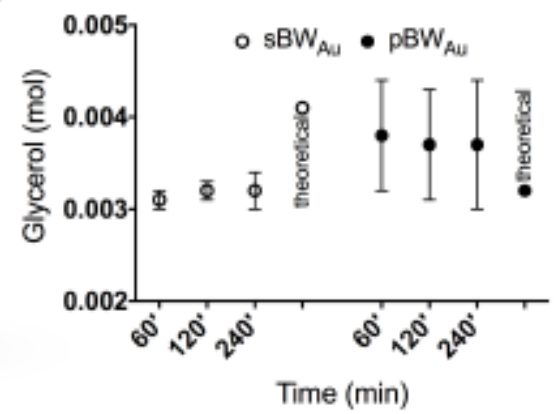

C



Figure 7
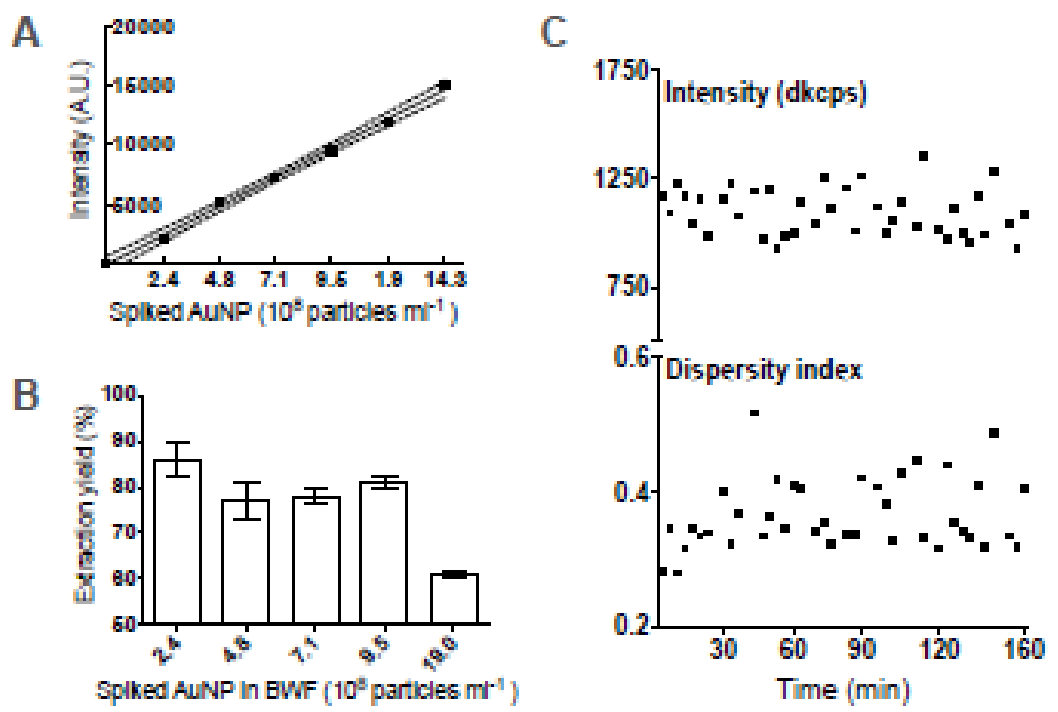

Figure 8
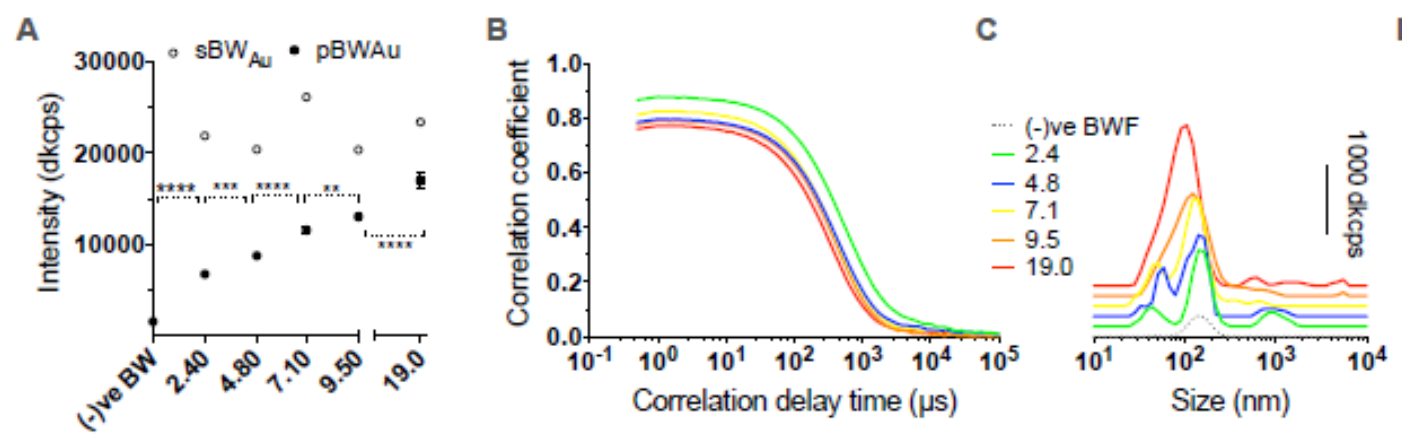

D

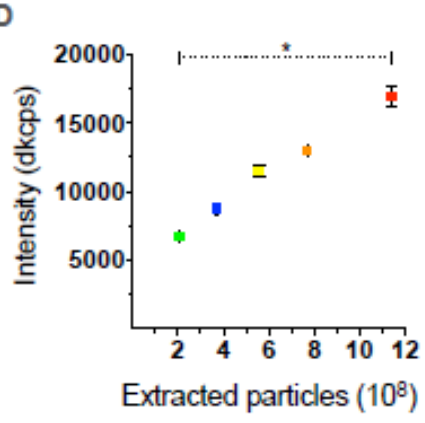


Figure 9

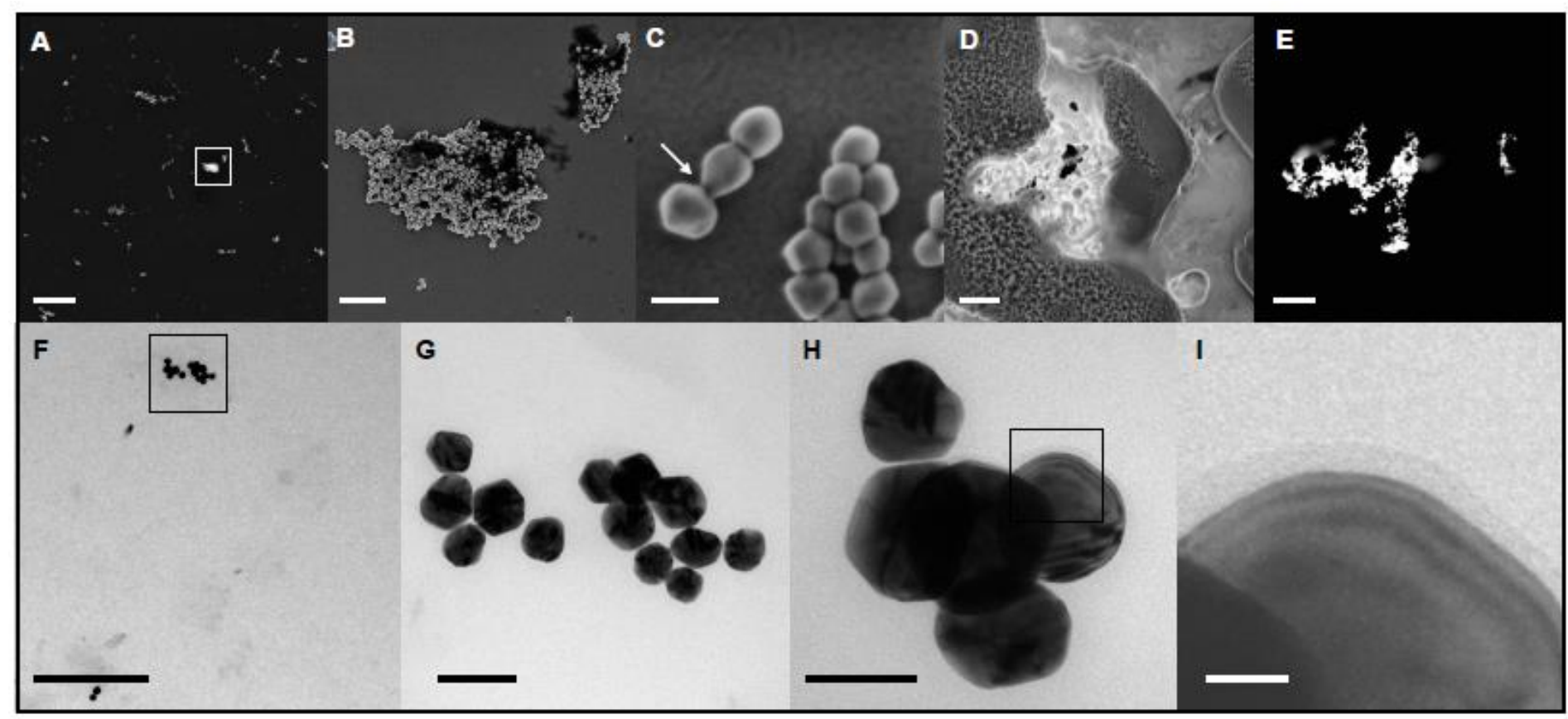

Figure 10




Figure 11
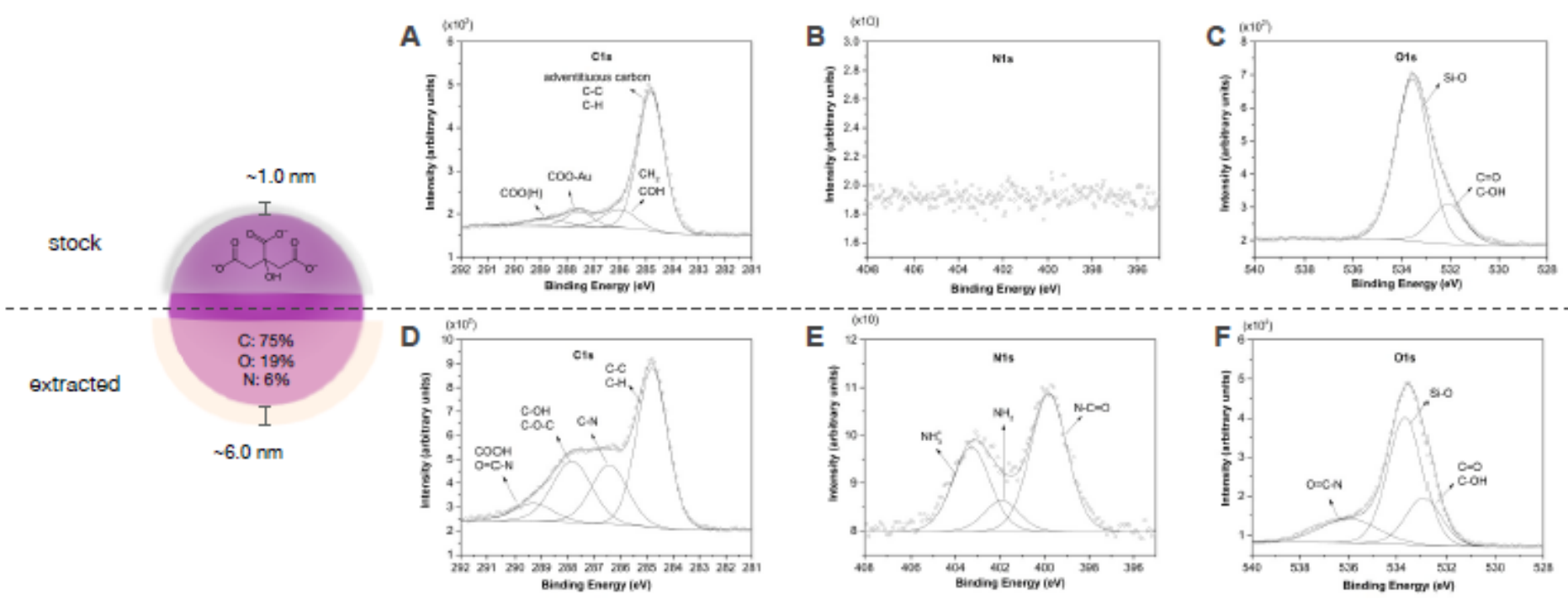

Figure 12


D

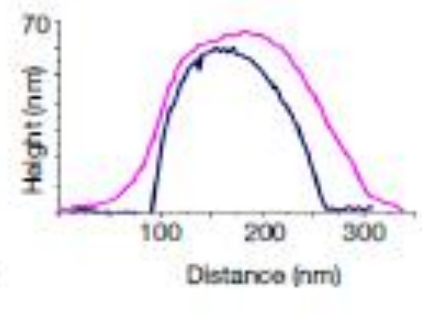




\section{SUPPLEMENTARY INFORMATION}

Supporting File S1: Derivation of formula for the extent of interdiffusion; calculation of the diffusion coefficient; relationships between glycerol concentration and solution viscosity and density; Nernst equation for the calculation of reduction potential; calculation of effective particle density.

\section{Formula for the extent of interdiffusion $\mathrm{d}$}

To derive the formula for the extent of interdiffusion d inside the cushion at any given time $t$, it was assumed that at the fringes of diffusion, glycerol concentration (C) needed to be $\geq 97.5 \%$ of its initial value $\mathrm{Cgc}$. Under the described experimental conditions, the relation between the initial glycerol concentrations in the aliquot $(\mathrm{Cal})$ and the cushion $(\mathrm{Cgc})$ in Step II is $\mathrm{Cgc}=5$ Cal (due to glycerol carried over from Step I). Substituting these conditions on the solution to Fick's second law for interdiffusing liquids

$$
\begin{gathered}
\mathrm{C}(\mathrm{x}, \mathrm{t})=0.5(\mathrm{Cgc}+\mathrm{Cal})-0.5(\mathrm{Cgc}-\mathrm{Cal}) \operatorname{erf}\left(0.5 \mathrm{x} \mathrm{D}^{-1 / 2} \mathrm{t}^{-1 / 2}\right) \\
\text { it becomes } \\
0.975 \mathrm{Cgc}=0.5(\mathrm{Cgc}+\mathrm{Cgc} / 5)-0.5(\mathrm{Cgc}-\mathrm{Cgc} / 5) \operatorname{erf}\left(0.5 \mathrm{~d} \mathrm{D}^{-1 / 2} \mathrm{t}^{-1 / 2}\right) \\
\text { and eventually } \\
-\operatorname{erf}\left(0.5 \mathrm{~d} \mathrm{D}^{-1 / 2} \mathrm{t}^{-1 / 2}\right)=1.317 \\
\quad \text { or } \\
\mathrm{d}=2.634 \mathrm{D}^{1 / 2} \mathrm{t}^{1 / 2} \approx(7 \mathrm{Dt})^{1 / 2}
\end{gathered}
$$

\section{Calculation of the diffusion coefficient $\mathrm{D}$}

The diffusion coefficient D for Step II was calculated by observing the interdiffusion between $0.5 \mathrm{ml}$ of cushion and a mixture of $1 \mathrm{ml}$ BWF with $0.25 \mathrm{ml}$ cushion (to represent the amount of cushion carried over from Step I, as calculated by eq. 2), over the course of $\mathrm{t}=4 \mathrm{~h}$ at $0^{\circ} \mathrm{C}$. The extent of interdiffusion was the distance between the initial and final position of the interface as evaluated by the grayscale color profile of the smeared interface in ImageJ (Figure A). Assuming that $\mathrm{d}=(7 \mathrm{Dt})^{1 / 2}$, it was calculated that $\mathrm{D} \approx 4.410-11 \mathrm{~m}^{2} \mathrm{~s}^{-1}$. 


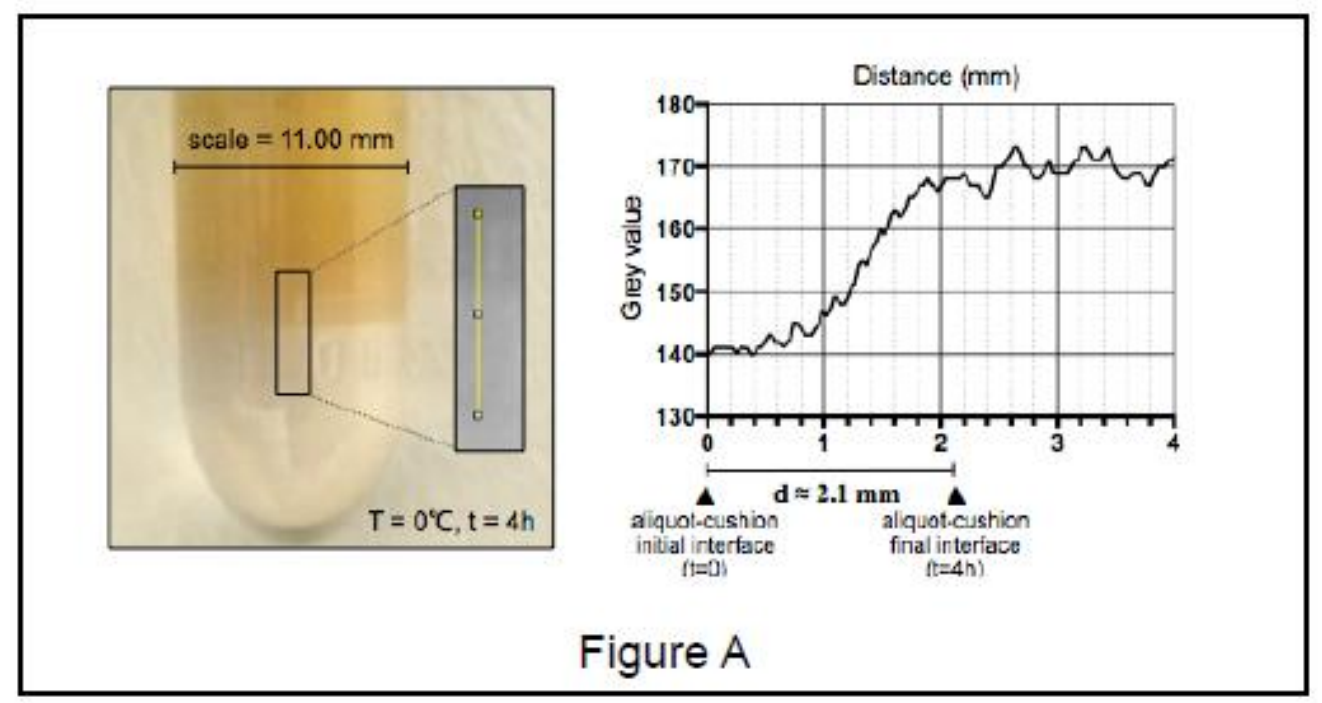

$\underline{\text { Relationship between solution density and glycerol concentration in glycerol-water solutions }}$ The density $\rho$ of glycerol-water solutions at $0^{\circ} \mathrm{C}$ at various glycerol concentrations was calculated based on a linear regression extrapolated by data found in the Handbook of Chemistry and Physics [1], presented in Figure B.

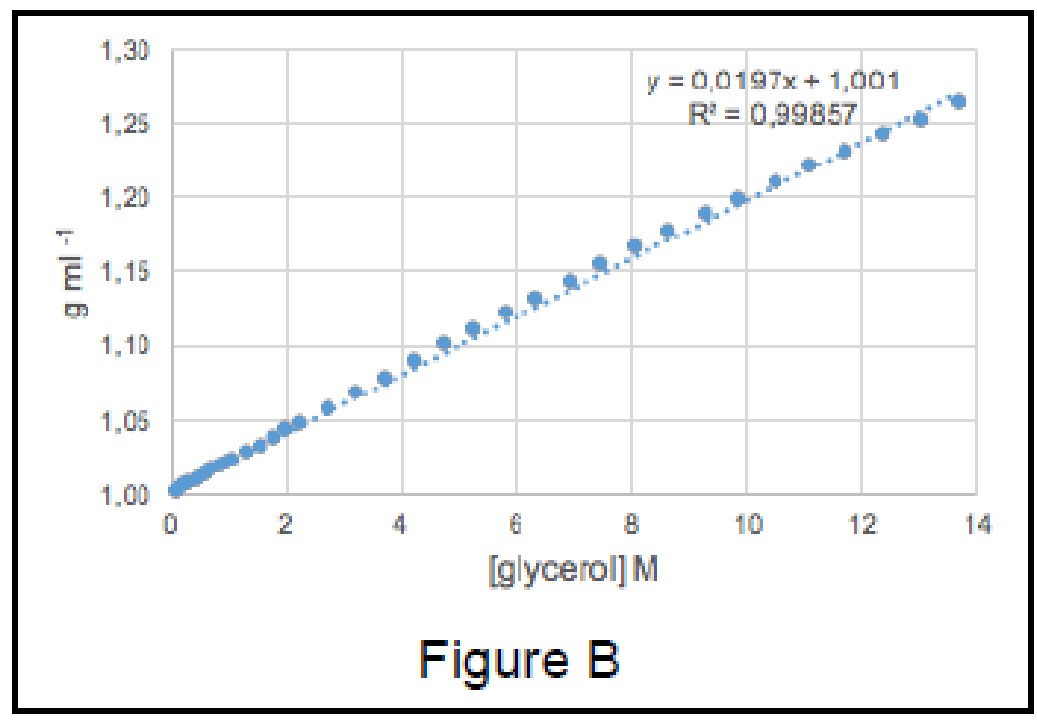

$\underline{\text { Relationship between solution viscosity and glycerol concentration in glycerol-water solutions }}$ Viscosity $\eta$ was calculated according to the formula proposed by Cheng [2]:

$$
\eta=\exp (\text { alpha* } \log (0.00173)) * \exp ((1-\text { alpha }) * \log (10.693))
$$

where

alpha $=(1-$ C_mass $)+\left(\alpha^{*} \beta^{*}\right.$ C_mass $*\left(1-C \_\right.$mass $\left.)\right) /\left(\alpha^{*}\right.$ C_mass $+\beta^{*}(1-$ C_mass $\left.)\right)$,

C_mass is the mass fraction of glycerol, and $\alpha, \beta$ are temperature-based parameters, with $\alpha=$ 0.705 and $\beta=2.045$ for $\mathrm{T}=0^{\circ} \mathrm{C}$. 
Nernst equation for the reduction potential of blank BWF

$$
\mathrm{E}_{\mathrm{ClO}^{-}}=\mathrm{E}^{0} \mathrm{ClO}^{-}-\left(\mathrm{RT} / \mathrm{n}_{\mathrm{e}^{-}} \mathrm{F}\right) \ln \left(\left[\mathrm{Cl}^{-}\right] /\left[\mathrm{ClO}^{-}\right]\right)
$$

where $\mathrm{E}_{\mathrm{ClO}}-$ is the half-cell oxidation potential of $\mathrm{ClO}^{-}, \mathrm{E}^{0} \mathrm{ClO}^{-}$is the standard half-cell oxidation potential of $\mathrm{ClO}^{-}$in an alkaline solution, $\mathrm{R}$ is the universal gas constant $\left(8.3 \mathrm{~J} \mathrm{~K}^{-1} \mathrm{~mol}^{-1}\right), \mathrm{T}$ is the temperature in kelvin, $\mathrm{F}$ is the Faraday constant $\left(9.6104 \mathrm{C} \mathrm{mol}^{-1}\right), \mathrm{n}_{\mathrm{e}}$ is the number of moles of electrons transferred in the half-cell reaction, and $\left[\mathrm{Cl}^{-}\right],\left[\mathrm{ClO}^{-}\right]$are the molar concentrations of $\mathrm{Cl}^{-}$and $\mathrm{ClO}^{-}$according to the nominal concentrations of saline and $\mathrm{NaOCl}$ solutions.

\section{Effective density of AuNP}

The effective density $\rho_{\mathrm{ED}}$ of suspended AuNP in BWF was roughly estimated as $17.055 \mathrm{~g} \mathrm{~cm}^{-3}$ using the Sterling equation [3]:

$$
\rho_{\mathrm{ED}}=\left(1-\varepsilon_{\alpha}\right) \rho_{\mathrm{p}}+\varepsilon_{\alpha} \rho_{\mathrm{BWF}}
$$

where $\rho_{\mathrm{p}}$ is the primary particle nominal density $\left(19.3 \mathrm{~g} \mathrm{~cm}^{-3}\right), \rho_{\mathrm{BWF}}$ is the BWF density, numerically equal to its relative density $\left(1.094 \mathrm{~g} \mathrm{~cm}^{-3}\right)$, and $\varepsilon_{\alpha}$ is the AuNP agglomerate porosity:

$$
\varepsilon_{\alpha}=1-\left(\mathrm{d}_{\mathrm{H}} \mathrm{dP}^{-1}\right)^{\mathrm{DF}-3}
$$

where $d_{H}$ is the AuNP agglomerate Z-average size measured by DLS $(74.8 \mathrm{~nm}), d_{P}$ is the smallest primary particle diameter observed by TEM $(45 \mathrm{~nm})$, and DF is a theoretical fractal dimension, equal to 2.3. Since it was impossible to calculate the agglomerate Z-average size in BWF, its value in DPBS was used instead. Also, the Sterling equation requires the particle diameter as determined by the Brunauer Emmet Teller method, but since AuNP were directly prepared in suspension, their primary particle size by TEM was used instead. Finally, the DF value was the one suggested by Deloid et al. for AuNP in cell culture media [4]. The estimated $\rho_{\text {ED }}$ was used in conjunction with the smallest observed primary particle diameter $d_{P}(45 \mathrm{~nm})$ so that the model returns the most conservative values regarding the required duration of the centrifugation in Step II.

1. Haynes WM: CRC handbook of chemistry and physics. CRC press; 2014.

2. Cheng N-S: Formula for the viscosity of a glycerol-water mixture. Industrial \& engineering chemistry research 2008, 47:3285-3288.

3. Cohen JM, Teeguarden JG, Demokritou P: An integrated approach for the in vitro dosimetry of engineered nanomaterials. Part Fibre Toxicol 2014, 11:20.

4. DeLoid G, Cohen JM, Darrah T, Derk R, Rojanasakul L, Pyrgiotakis G, Wohlleben W, Demokritou P: Estimating the effective density of engineered nanomaterials for in vitro dosimetry. Nat Commun 2014, 5:3514. 


\section{Supporting Figure S2: Sample preparation for field emission scanning electron microscopy (FESEM) and back-scatter electron microscopy (BSE)}

A, B) A mica sheet is deposited on the aluminium stub where it can be secured with a small piece of double-sided adhesive tape.

C) The stub was then placed in a Gatan 682 precision coating system and a $50 \mathrm{~nm}$ highresolution carbon layer was deposited on top of the mica sheet.

D) The coated mica sheet was electrically connected to the second rim of the stub with conductive tape.

E) Sample drops (1-2 $\mu \mathrm{l})$ were deposited on the coated surface and were left to dry with the stub positioned horizontally and protected by environmental air.

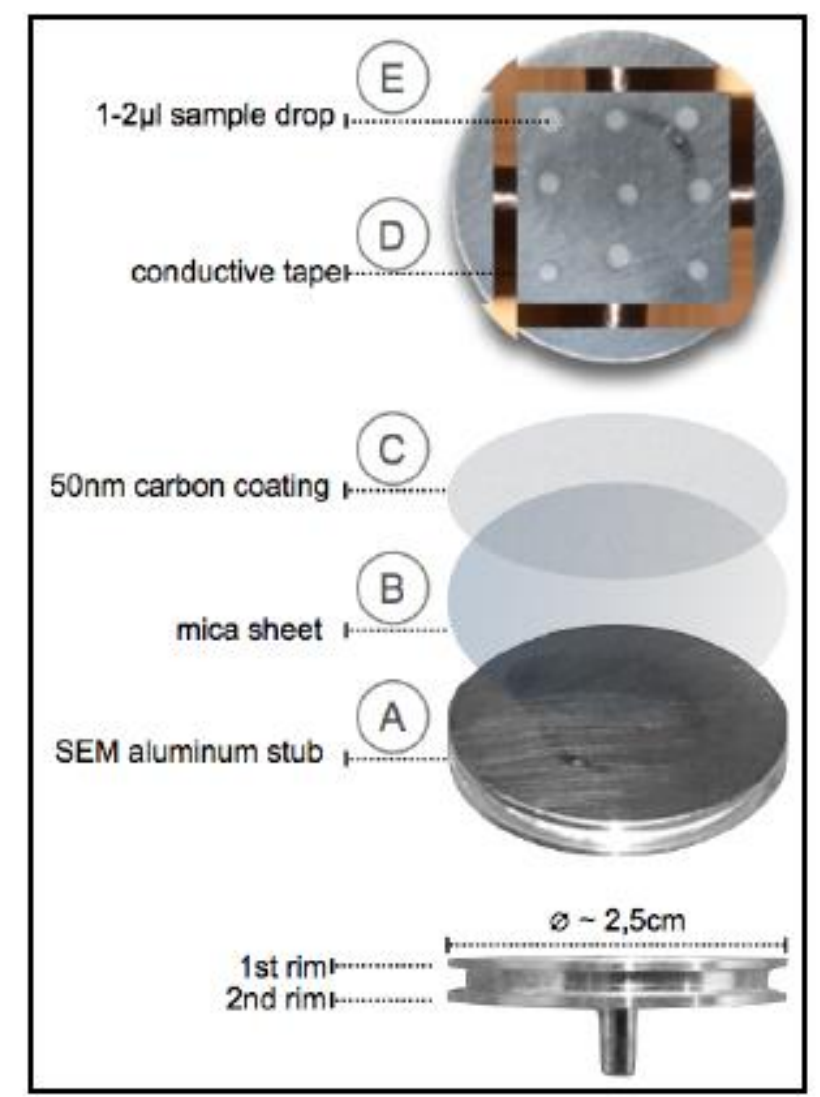




\section{Supporting Table S3: Dissolution of nanoparticles in blank BWF}

The reduction potential and $\mathrm{pH}$ of blank BWF (50\% v/v of $0.0183 \mathrm{M}$ Na-hypochlorite in 0.9 $\mathrm{w} / \mathrm{v} \mathrm{NaCl}$ ) were calculated at $0.87 \mathrm{~V}$ and 11.4 , respectively. These values are expected to become lower in the presence of biomolecules found in real BWFs. The compositional stability of several prominent nanoparticles was assessed in blank BWF. Specifically, citrate-capped Au and $\mathrm{Ag}, \mathrm{Al}_{2} \mathrm{O}_{3}, \mathrm{Fe}$, amorphous $\mathrm{SiO}_{2}, \mathrm{TiO}_{2}$, and $\mathrm{ZnO}$ nanoparticle suspensions at varied concentrations were added to $1.00 \mathrm{ml}$ of blank BWF in $2 \mathrm{ml}$ Protein LoBind tubes (Eppendorf®) and vortexed for a few seconds. The tubes were then sealed and stored at $4{ }^{\circ} \mathrm{C}$ for a minimum of 1 week, protected from light. To measure the dissolved particle fraction, $0.40 \mathrm{ml}$ of each dispersion was transferred in $100 \mathrm{kDa}$ Amicon ${ }^{\circledR}$ Ultra- 0.5 centrifugal filter tubes of a nominal molecular weight cut-off of $100 \mathrm{kDa}$ (Merck MilliporeTM) and centrifuged at $14000 \times \mathrm{g}$ in a fixed-angle rotor (Eppendorf® MiniSpin PlusTM centrifuge, ThermoFisher ScientificTM) for $10 \mathrm{~min}$ at room temperature. The filtrates $(\approx 0.4 \mathrm{ml})$ were then added in $9.60 \mathrm{ml}$ of $2.0 \mathrm{M} \mathrm{HCl}$ and the concentration of dissolved metals were measured by ICP-OES against a standard multielement solution in 5\% w/w HNO3 (SCP Science). Results indicated that $\mathrm{Al}_{2} \mathrm{O}_{3}$, citrate-capped $\mathrm{Au}, \mathrm{Fe}, \mathrm{TiO}_{2}$, and $\mathrm{ZnO}$ were minimally or not dissolved at all; citrate-capped Ag nanoparticles were completely dissolved, most probably by the formation of $\mathrm{AgCl}$; amorphous $\mathrm{SiO}_{2}$ were almost completely dissolved (71\%).

Table S3 Primary particle size and dissolution in blank BWF of a panel of prominent nanoparticles.

\begin{tabular}{|c|c|c|c|c|c|c|c|}
\hline $\begin{array}{l}\text { core } \\
\text { composition }\end{array}$ & Ag & $\mathrm{Al}_{2} \mathrm{O}_{3}$ & $\mathbf{A u}$ & $\mathbf{F e}$ & $\mathrm{SiO}_{2}$ & $\mathrm{TiO}_{2}$ & $\mathrm{ZnO}$ \\
\hline $\begin{array}{l}\text { size range }(\mathrm{nm}) \\
\text { *nominal }\end{array}$ & $36-44$ & $30-60$ & $61-73$ & $35-45$ & $22 *$ & $\leq 150^{*}$ & $\leq 100^{*}$ \\
\hline \% dissolution & 106.3 & 0.6 & Non & 0.0 & 71.0 & Non & 6.0 \\
\hline S.D. & 1.9 & 0.4 & detectable & 0.0 & 1.0 & detectable & 0.0 \\
\hline
\end{tabular}


Supporting Figure S4: Application of the extraction method on fetal bovine serum spiked with AuNP

The extraction method was performed on undiluted fetal bovine serum spiked with AuNP at $2.50,5.00,7.50$, and $10.00 \%$ of the stock particle concentration. In this medium, protein molecules adsorb on the particles' surface but have been shown to cause only low agglomeration [1]. Extraction yields were similar to those achieved in BWF, except for the highest concentration where less agglomeration in FBS allowed for even better yields. AuNP were spread throughout the cushion and allowed for the plasmon resonance effect to take place (image below). Some pelleting did still occur at the highest particle concentration, but to less extent when compared to BWF (inset of image below). The extraction yield as measured by ICP-OES ranged from 70 to $86 \%$ attaining similar levels to BWF, but performing significantly better at the highest concentration due to less agglomeration.

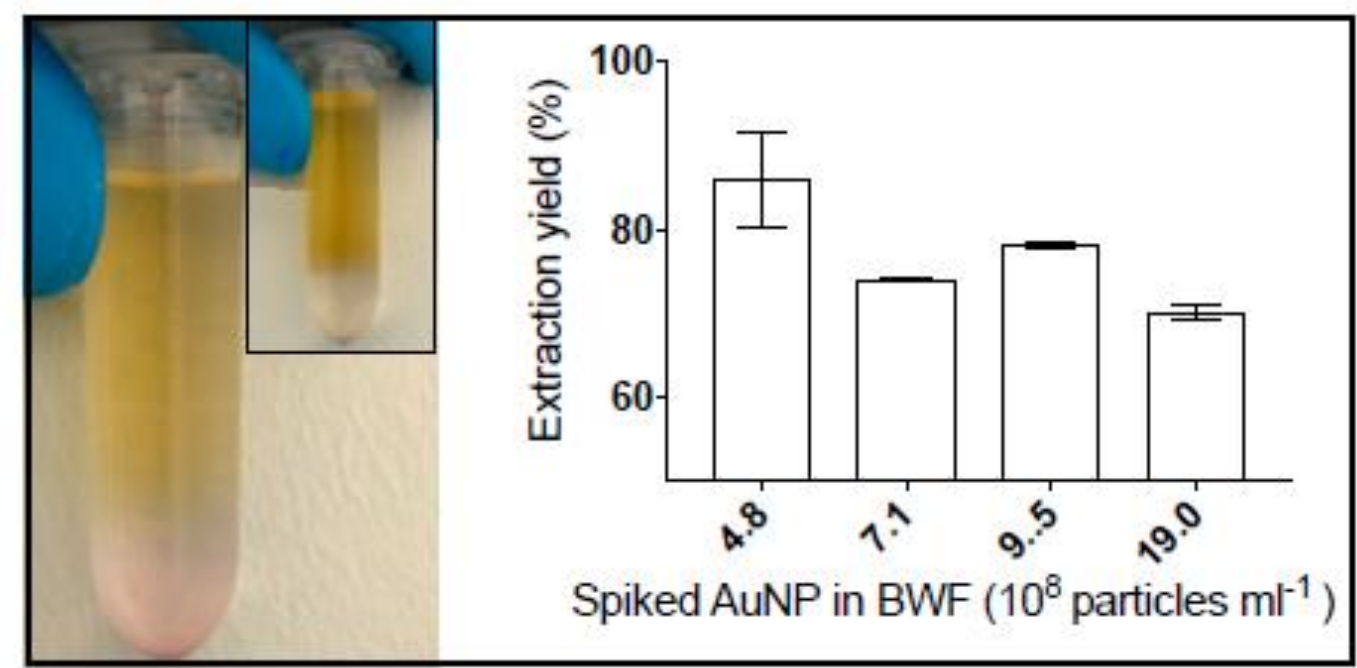

1. Casals E, Pfaller T, Duschl A, Oostingh GJ, Puntes V: Time evolution of the nanoparticle protein corona. ACS Nano 2010, 4:3623-3632. 
Supporting File S5: Application of the extraction method on bronchial washing fluids from two patients

The method was applied on bronchial washing fluids from two patients (A and B) with symptoms of infiltrative pulmonary diseases. A summary of their clinical data is presented in Table 1; ICP-OES and DLS data are summarized in Figures A and B, respectively; optical microscopy, BSE, and in-lens FESEM images along with particle perimeter analysis by ImageJ are presented in Figure C. Despite the presented data, a full mineralogical analysis of the extracted would require additional experiments, e.g. high-resolution EDX and TEM measurements in order to determine the chemical composition and crystal structure of the visualised particles

Table 1 Clinical data summary collected from patients A and B

\begin{tabular}{|c|c|c|c|c|c|c|}
\hline Tobacco & Residential \\
use & environment & $\begin{array}{c}\text { Professional } \\
\text { exposure }\end{array}$ & $\begin{array}{c}\text { Nanoparticle } \\
\text { exposure }\end{array}$ & $\begin{array}{c}\text { Symptoms } \\
\text { \& findings }\end{array}$ & Diagnosis \\
\hline A & Former & Rural & $\begin{array}{c}\text { Asbestos } \\
(15 \text { years })\end{array}$ & $\begin{array}{c}\text { Perfume } \\
\text { Hydration } \\
\text { creme }\end{array}$ & $\begin{array}{c}\text { Acute } \\
\text { CT scan: } \\
\text { lung shadow }\end{array}$ & $\begin{array}{c}\text { Drug-induced } \\
\text { lung disease }\end{array}$ \\
\hline B & Nonsmoker & Rural & - & $\begin{array}{c}\text { Perfume } \\
\text { Hydration } \\
\text { creme } \\
\text { Toothpaste }\end{array}$ & $\begin{array}{c}\text { Chronic } \\
\text { CT scan: } \\
\text { micronodule }\end{array}$ & $\begin{array}{c}\text { Lymphangitis } \\
\text { carcinomatosa }\end{array}$ \\
\hline
\end{tabular}
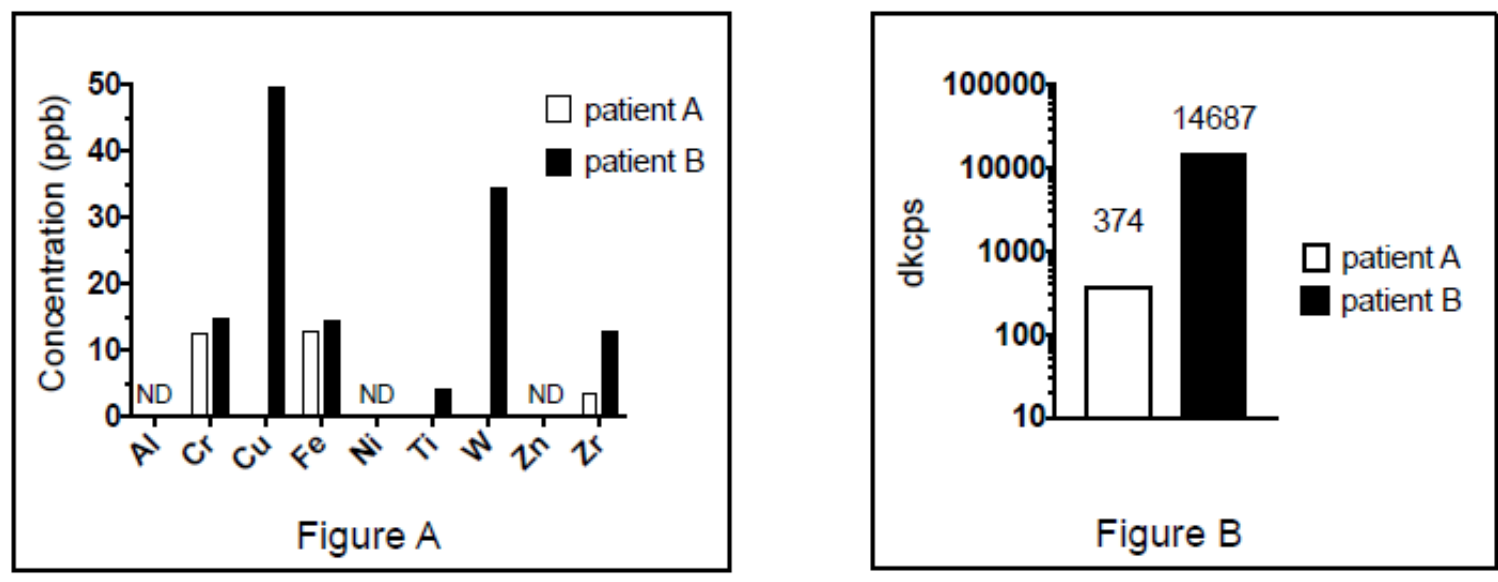

Metal (Al, Co, Cr, Cu, Fe, Ni, Ti, W, Zn, Zr) concentrations (Figure A) and dkcps signals (Figure B) of the nanoparticle-containing fraction of patient B were generally stronger than the respective signals retrieved from patient A, possibly indicating a larger nanoparticle loads. 


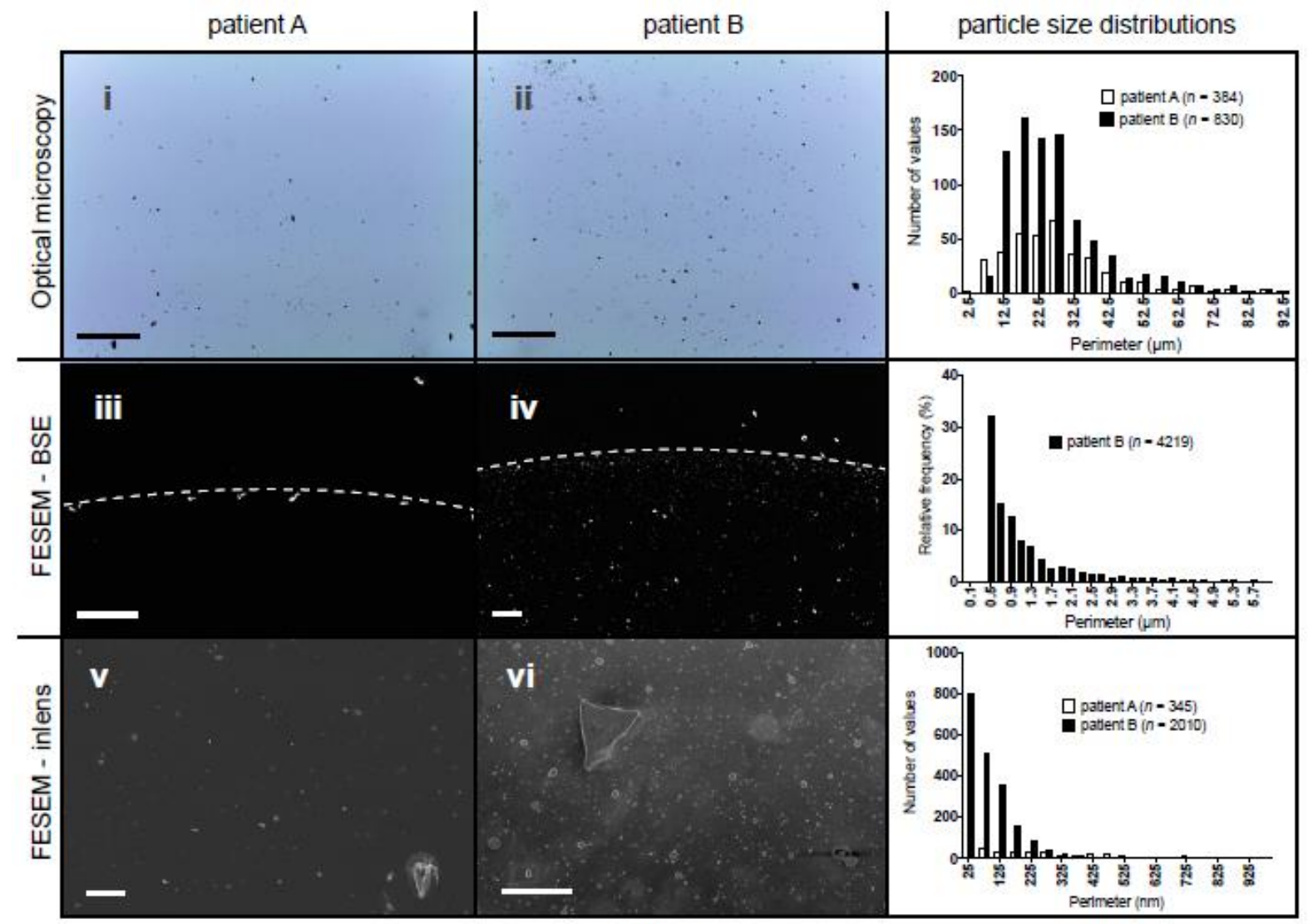

Figure $\mathrm{C}$

i, ii) Optical microscopy could only resolve coarse particles extracted after Step I.

iii, iv) BSE images of smaller particles extracted after Step II: this technique detects electronically dense and/or protruding particles; dashed white lines are visual aids along the borders of the dried sample droplets where particles have been deposited due to the coffee ring effect effect. Interestingly, very large particles (measuring several microns in perimeter) are largely absent, indicating that they were successfully extracted in Step I.

v, vi) Higher magnification, in-lens FESEM imaging is very sensitive to morphological changes of the examined surface and revealed a markedly denser submicron and nanosized particle population for patient $\mathrm{B}$.

Scale-bars: i, ii, $50 \mu \mathrm{m}$; iii, iv, $10 \mu \mathrm{m}$; v, vi, $2 \mu \mathrm{m}$.

\section{particle size distributions:}

Coarse particle populations presented similar size distributions under optical microscopy, but patient $\mathrm{B}$ carried more than twice particles than patient A. BSE imaging detected mainly submicron-sized particles in patient B while very few particles (12) were detected in patient A. Finally, Under in-lens FESEM imaging, patient B carried $\times 6$ timed more nanoparticles than patient A. 


\section{Supporting File S6: Calculation of organic shell thickness and composition by XPS}

In a core-shell system consisting of a spherical nanoparticle with a thin nanometric overlayer, the thickness of the shell can be calculated using the intensities ratio of photoelectrons coming from the shell and photoelectrons coming from the core, weighted by intensities of pure shell and core materials [1]. In our case, this ratio gives the following equation:

$$
A=\frac{I_{x} I_{A u 4 f}^{\infty}}{I_{A u 4 f} I_{x}^{\infty}}
$$

where $x$ is an element constituting the organic shell $(\mathrm{C}, \mathrm{O}$ or $\mathrm{N})$. The method takes also into account the travelling path of photoelectrons with two parameters, $B$ and $C$, corresponding to ratios of attenuation length $\mathrm{L}_{\mathrm{a}, \mathrm{b}}$ of photoelectrons coming from core level a and travelling through material $b$. The two ratios are defined as:

$$
\begin{aligned}
& B=\frac{L_{A u 4 f, \text { shell }}}{L_{x, \text { shell }}} \\
& C=\frac{L_{A u 4 f, \text { shell }}}{L_{\text {Au } 4 \text { f, core }}} \quad \text { eq. } 2
\end{aligned}
$$

The values of La,b, which are reported in Table 1, were estimated according to equation S4 from the paper of Seah [2] and as demonstrated in the work of Belsey et al. [3]. According to Shard, the shell thickness TNP for a nanoparticle with a core radius R can be obtained with a precision of $4 \%$ using the following set of equations:

$$
\begin{array}{ll}
T_{N P}=\frac{T_{R \sim 1}+\beta T_{0}}{1+\beta} & \text { eq. } 4 \\
T_{0}=R\left[(A B C+1)^{1 / 3}-1\right] & \text { eq. } 5 \\
T_{R \sim 1}=\frac{T_{R \rightarrow \infty} R}{R+\alpha} & \text { eq. } 6 \\
\alpha=\frac{1.8}{A^{0.1} B^{0.5} C^{0.4}} & \text { eq. } 7 \\
\beta=\frac{0.13 \alpha^{2.5}}{R^{1.5}} & \text { eq. } 8
\end{array}
$$




$$
T_{R \rightarrow \infty}=\frac{0.74 A^{3.6} \ln (A) B^{-0.9}+4.2 A B^{-0.41}}{A^{3.6}+8.9} \quad \text { eq. } 9
$$

It is worth noting that, $R$ and $T_{N P}$ are expressed in units of $L A u 4 f$,shell.

Because intensities from pure organic shell material cannot be obtained, equation 10 from the work of Belsey et al. was used, where $[\mathrm{x}]$ and $[\mathrm{Au}]$ are the atomic percentage of the elements $\mathrm{x}$ and $\mathrm{Au}$ considering that these elements are homogeneously distributed in all the depth of analysis, $\mathrm{cx}$ the atomic fraction of the element $\mathrm{x}$ in the organic shell, and $f$ an additional factor used to compensate for the different attenuation length, density, and intrinsic loss processes between the gold core and organic shell:

$$
A_{x}=f \frac{[x]}{[A u] c_{x}}
$$

In this work, we make the assumption that the value of $f$ is close to the one from the work of Belsey et al., which gives 0.56 . As described in their work, a shell thickness $T_{N P}$ is calculated for each element $x$ present in the shell. With the constraint that the sum of all $c_{x}$ equals 1 , the result is computed iteratively varying the value of $c_{x}$ until the same $T_{N P}$ is found for each ratio $A_{x}$.

The computation of $T_{N P}$ was carried out considering an averaged core radius of $30 \mathrm{~nm}$ for the nanoparticles. Oxygen and carbon were considered as the elements forming the organic shell in the case of stock gold nanoparticles while oxygen, carbon and nitrogen were considered for the extracted gold nanoparticles. The calculation was made using the intensities of the photoelectron peaks O1s, C1s and N1s for the shell while for the core signal the Au4f peak was chosen.

For the O1s peak, the intensity of the component $\mathrm{Si}-\mathrm{O}$ assigned to the silicon wafer native oxide was excluded and subtracted from the total intensity of O1s. For the C1s peak, in the case of stock AuNP, the intensity of the component assigned to adventitious carbon on the silicon wafer was also excluded. Using these assumptions, the atomic fraction of oxygen and carbon calculated for the organic layer on the stock nanoparticles with the iterative procedure were estimated respectively to 0.53 and 0.47 which is in accordance to the chemical formula of the citrate molecule. For the extracted nanoparticles, it is not possible to distinguish the adventitious carbon from the $\mathrm{C}-\mathrm{C}$ and/or $\mathrm{C}-\mathrm{H}$ chemical bindings in the proteins molecules. However, since we consider that the adventitious carbon comes mainly from the area of silicon wafer not covered by AuNP, we attempt to find an expression of the intensity of adventitious carbon for 
the sample with extracted nanoparticles $I_{C 1 s}^{\text {adv C/extractedNP }}$ in function of the one for the sample with stock nanoparticles $I_{C l s}^{a d v C / s t o c k N P}$, Equation 11 shows that the ratio of the intensity of Si2p for a silicon reference sample $I_{S i 2 p}^{r e f}$ and for the sample with stock AuNP can be written as a linear relationship with $1 / \cos \theta$ where $\theta$ is the escape angle of the photoelectrons with respect to the normal of the sample. This expression allows estimating the thickness of the adventitious carbon layer $\mathrm{d}^{\text {advC/stockNP }}$ and the fraction of the wafer not covered by the nanoparticles $\sigma^{\text {advC/stockNP }}$. The same type of relationship can be written for the ratio of the intensity of Si2p for the sample with stock AuNP and the sample with extracted AuNP as shown in equation 12. This second expression allows calculating the adventitious carbon layer $\mathrm{d}^{\mathrm{advC} / \mathrm{stockNP}}$ and the fraction of the wafer not covered by the nanoparticles $\sigma^{\text {advC/stockNP }}$ for the sample with extracted AuNP.

$$
\begin{gathered}
\frac{d^{a d v C / s t o c k N P}}{L_{S i 2 p, a d v C} \cos \theta}+\ln \left(\sigma^{a d v C / \text { stockNP }}\right)=\ln \left(\frac{I_{S i 2 p}^{\text {ref }}}{I_{S i 2 p}^{\text {stockNP }}}\right) \\
\frac{d^{a d v C / \text { extracted } N P}-d^{a d v C / \text { stockNP }}}{L_{S i 2 p, a d v C} \cos \theta}-\ln \left(\frac{\sigma^{\text {advC/extractedNP }}}{\sigma^{\text {advC/stockNP }}}\right)=\ln \left(\frac{I_{S i 2 p}^{\text {stockNP }}}{I_{S i 2 p}^{\text {extracted } N P}}\right)
\end{gathered}
$$

Figure $\mathrm{C}$ presents the adjustment of these linear equations with XPS measurements at different photoelectron takeoff angles. The fitting results allows for the calculation of the thicknesses and coverages of the adventitious carbon layer for both stock and extracted AuNP, using the attenuation length in Table 1 for $\mathrm{LSi2p}_{\mathrm{S} \text {,advC }}$ which are presented in Table 2 . The intensity of the adventitious carbon component in the C1s peak for the sample with extracted AuNP can be described by the following equation:

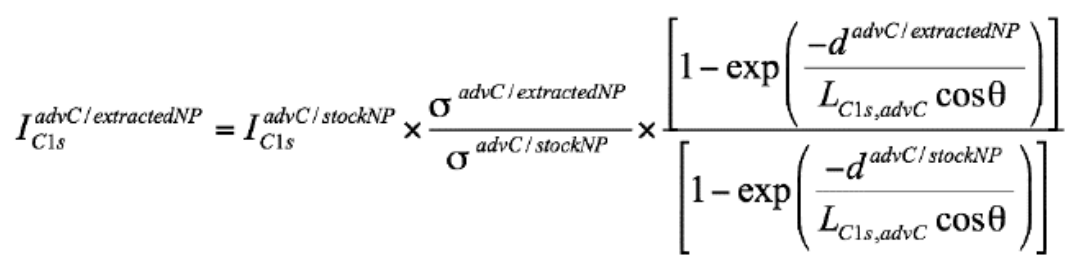

Using the values in Table 2, the attenuation length $\mathrm{L}_{\mathrm{C} 1 \mathrm{~s}, \mathrm{advC}}$ given in Table 1 and for a takeoff angle $\theta$ of $50^{\circ}$, this intensity can be written as:

$$
I_{C 1 s}^{a d v \text { Cextracted } N P}=1.151 \times I_{C 1 s}^{a d v C / \text { stockNP }}
$$

This intensity was then subtracted from the intensity of the C1s peak in order to calculate the thickness and composition of the organic shell for the extracted AuNP. 
Table 1 Attenuation length calculated using the equation S4 in the paper of for electrons coming from different core levels and travelling through a system consisting of gold nanoparticle core with an organic shell.

\begin{tabular}{|c|c|c|}
\hline & L (organic shell), $\mathrm{nm}$ & L (Au core), $\mathrm{nm}$ \\
\hline $\mathrm{C} 1 \mathrm{~s}$ & 3.32 & 1.17 \\
\hline $\mathrm{O} 1 \mathrm{~s}$ & 2.74 & 0.99 \\
\hline $\mathrm{N} 1 \mathrm{~s}$ & 3.05 & 1.09 \\
\hline $\mathrm{Au} 4 \mathrm{f}$ & 3.79 & 1.33 \\
\hline $\mathrm{Si} 2 \mathrm{p}$ & 3.75 & $\sim$ \\
\hline
\end{tabular}

Figure C Adjustment of the expression given by equations 11 and 12 with experimental XPS measurements at several photoelectrons collection angles of the intensity of Si2p peak for a reference sample, the sample with stock Au nanoparticles and the sample with extracted $\mathrm{Au}$ nanoparticles.

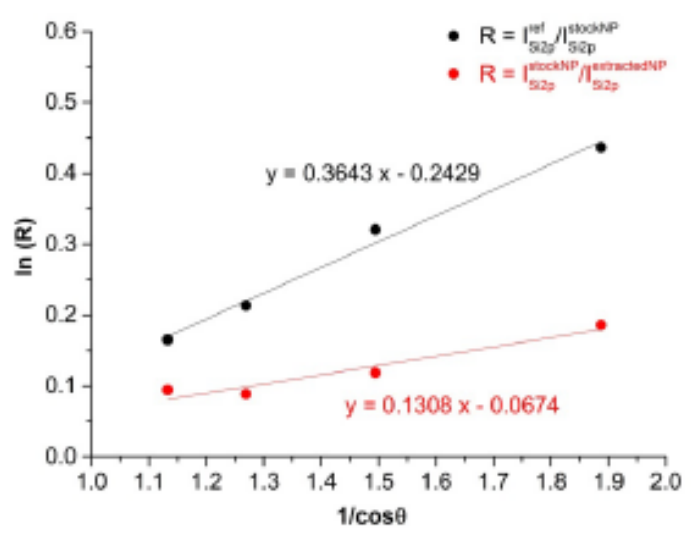

Table 2 Thicknesses and coverage of the adventitious carbon layer on the silicon wafer substrate for the sample with stock and extracted nanoparticles.

\begin{tabular}{|c|c|c|}
\hline sample & $\mathrm{d}^{\mathrm{advC}}(\mathrm{nm})$ & $\sigma^{\mathrm{advC}}$ \\
\hline Si/stock Au nanoparticles & 1.36 & 0.78 \\
\hline Si/extracted Au nanoparticles & 1.85 & 0.73 \\
\hline
\end{tabular}

1. Shard AG: A straightforward method for interpreting XPS data from core-shell nanoparticles. The Journal of Physical Chemistry C 2012, 116:16806-16813.

2. Seah M: Simple universal curve for the energy-dependent electron attenuation length for all materials. Surface and Interface Analysis 2012, 44:1353-1359.

3. Belsey NA, Shard AG, Minelli C: Analysis of protein coatings on gold nanoparticles by XPS and liquid-based particle sizing techniques. Biointerphases 2015, 10:019012. 